\title{
Fairness and Incentive Considerations in Energy Apportionment Policies
}

Ekhiotz J on Vergara Alonso, Simin Nadjm-Tehrani and Mikael Asplund

The self-archived version of this journal article is available at Linköping University Electronic Press:

http:/ / urn.kb.se/ resolve?urn=urn:nbn:se:liu:diva-138088

N.B.: When citing this work, cite the original publication.

Vergara Alonso, E. J ., Nadjm-Tehrani, S., Asplund, M., (2016), Fairness and Incentive Considerations in Energy Apportionment Policies, ACM Transactions on Modeling and Performance Evaluation of Computing Systems, 2(1). https:// dx.doi.org/ 10.1145/ 2970816

Original publication available at:

https:/ / dx.doi.org/ 10.1145/2970816

Copyright: ACM 


\title{
Fairness and Incentive Considerations in Energy Apportionment Policies
}

\author{
EKHIOTZ JON VERGARA, SIMIN NADJM-TEHRANI and MIKAEL ASPLUND, \\ Linköping University
}

\begin{abstract}
The energy consumption of a system is determined by the system component usage patterns and interactions between the coexisting entities and resources. Energy accounting plays an essential role to reveal the contribution of each entity to the total consumption and for energy management. Unfortunately, energy accounting inherits the apportionment problem of accounting in general, which does not have a general single best solution. In this paper we leverage cooperative game theory commonly used in cost allocation problems to study the energy apportionment problem, i.e., the problem of prescribing the actual energy consumption of a system to the consuming entities (e.g., applications, processes or users of the system).

We identify five relevant fairness properties for energy apportionment and present a detailed categorisation and analysis of eight previously proposed energy apportionment policies from different fields in computer and communication systems. In addition, we propose two novel energy apportionment policies based on cooperative game theory which provide strong fairness notion and a rich incentive structure. Our comparative analysis in terms of the identified five fairness properties as well as information requirement and computational complexity shows that there is a trade-off between fairness and the other evaluation criteria. We provide guidelines to select an energy apportionment policy depending on the purpose of the apportionment and the characteristics of the system.
\end{abstract}

Categories and Subject Descriptors: C.4 [Performance of Systems] Modeling techniques

General Terms: Design, Performance, Management

Additional Key Words and Phrases: energy apportionment, energy accounting, cooperative game theory, energy management

ACM Reference Format:

Vergara, E. J. and Nadjm-Tehrani, S. and Asplund, M. 2015. Fairness and Incentive Considerations in Energy Apportionment Policies. TOMPECS 2, 1, Article 2 (November 2016), 27 pages.

DOI $=10.1145 / 2970816$ http://doi.acm.org/10.1145/2970816

\section{INTRODUCTION}

Energy consumption and its management is clearly a challenge in computing and communication system design. Energy consumption is obviously of paramount importance for battery powered devices, but energy efficiency has also become essential for plugged systems due to core drivers such as sustainability and operational expenses.

Author's address: E. J. Vergara, email: ekhiotz.vergara@liu.se; Simin Nadjm-Tehrani, simin.nadjm-tehrani@liu.se; Mikael Asplund, email: mikael.asplund@liu.se; Department of Computer and Information Science, Linköping University, Sweden

Permission to make digital or hard copies of part or all of this work for personal or classroom use is granted without fee provided that copies are not made or distributed for profit or commercial advantage and that copies show this notice on the first page or initial screen of a display along with the full citation. Copyrights for components of this work owned by others than ACM must be honored. Abstracting with credit is permitted. To copy otherwise, to republish, to post on servers, to redistribute to lists, or to use any component of this work in other works requires prior specific permission and/or a fee. Permissions may be requested from Publications Dept., ACM, Inc., 2 Penn Plaza, Suite 701, New York, NY 10121-0701 USA, fax +1 (212) 869-0481, or permissions@acm.org.

(C) 2016 ACM 0000-0000/2016/11-ART2 $\$ 15.00$

DOI 10.1145/2970816 http://doi.acm.org/10.1145/2970816 
Even though it can be argued that energy is just another resource subject to common methods for resource management [Neugebauer and McAuley 2001], energy management poses its own challenges [Zeng et al. 2002a; Roy et al. 2011]. The energy consumption created by a resource usage is complex since (1) the amount of activity of an entity is often not proportional to the consumed energy, and (2) energy consumption does not necessarily correspond to the time interval in which the activity happened. Additionally, the coexisting accesses to shared resources by the different entities in the system aggravate the problem, which results in the actual consumption not being the sum of what the entities would consume using the resource alone.

Energy accounting is the procedure of quantifying, analysing and reporting the energy consumption of different entities or activities of interest in the system. Efficiently managing energy requires a detailed understanding of the level of resource consumption and energy accounting is a key medium to provide the needed transparency to the system. Energy accounting is also vital to evaluate the energy efficiency of entities in the system. There is evidence supporting the fact that energy awareness can aid to conserve energy [Petersen et al. 2007; Athukorala et al. 2014]. Unfortunately, apportionment is a hard problem of accounting in general which does not have a single best solution for all cases [Lemaire 1984], and energy accounting does not escape from this problem.

Selecting an apportionment method, i.e., a method to divide the shared energy consumption, entails a set of principles used to achieve some rational division. Thus, we call these methods energy apportionment policies referring to the rule that prescribes the consumed energy to each entity. Examples of entities are different applications using a certain resource, processes of interest or system users. We illustrate the apportionment problem with two instances in the context of mobile and wireless networks, but as we show later in our work the problem arises in multiple different contexts.

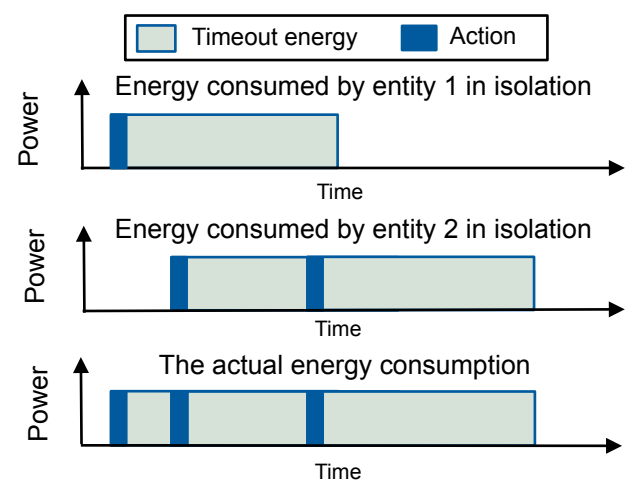

System states:

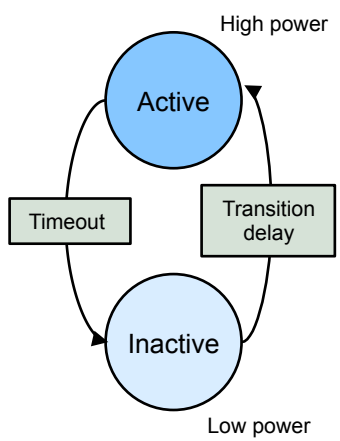

Fig. 1: Energy apportionment example for a common simplification of a system.

Fig. 1 exemplifies a simple apportionment case. The system is characterised by two states, active and inactive, where an entity can perform an activity only in the active state. After every activity a timeout is activated which keeps the system consuming energy in the active state for a while. The timeout is a power management mechanism that also serves responsiveness by avoiding the inactive to active state transition delay for subsequent activities. Many computing and communication components or systems show a similar behaviour, such as wireless interfaces [Balasubramanian et al. 2009; Vergara et al. 2014b; Pathak et al. 2012; Mittal et al. 2012], hard drives [Papathanasiou and Scott 2003], Secure Digital cards [Pathak et al. 2012] or even some electric appliances [Reinhardt et al. 2012a].

Fig. 1 shows the energy consumption of the entities if they were alone in the system (i.e., in isolation). The actual total consumption of the interface is not the sum of entities' consumption in isolation. How should we 
prescribe a share of the total energy consumption to the different entities in the system? Equally dividing the energy consumption would mean that entity 1 subsidises entity 2 . Would a proportional apportionment policy be better?

Another example is from a wireless sensor network scenario (also applicable to an Internet of Things context)[Fonseca et al. 2008; Kellner 2010], where determining the energy consumption of network nodes due to user defined activities (e.g., queries) is required. In this scenario, the total energy consumption of the system is determined by the sum of each node's consumption, but the contribution of each node to the total consumption is not necessarily its own consumption. A node contributes to other nodes' energy consumption by being part of the system (e.g., transmissions or beacons). Determining the contribution of each node and the consumption due to the user-defined activities requires a solution to the apportionment problem as well.

Selecting an apportionment policy is a fundamental choice for a system, not only it enables the assessment of the energy consumption of an entity in the system, but we show that it also establishes the "rules of the game" for the system which potentially influence the behaviour of the entities, e.g., by providing incentives. The latter aspect is related to fairness of the apportionment that is often hidden at first sight in an energy apportionment policy.

In our work we show that energy apportionment is a common problem in different areas of computer science, ranging over computing [Flinn and Satyanarayanan 1999b; Ryffel et al. 2009], data centres [Bertran et al. 2010], mobile devices [Zhong and Jha 2003; Mittal et al. 2012; Neugebauer and McAuley 2001; Pathak et al. 2012; Roy et al. 2011; Dong et al. 2014], wireless sensor networks [Sieber and Nolte 2013; Kellner 2010], and energy-efficient buildings [Hay and Rice 2009; Tsao et al. 2014; Thakur et al. 2014; Cheng et al. 2012]. Our work is the first recognising the common ground of the energy apportionment problem in these areas and builds a bridge to study the this problem through the lens of cooperative game theory. This provides excellent mathematical methods to allocate cooperative costs and evaluate the solutions already used in multiple real world practical problems [Fiestras-Janeiro et al. 2011; Moretti and Patrone 2008]. The additional contributions of our work are the following:

(1) Identify 5 relevant fairness criteria for energy apportionment. We show that providing incentives to improve the energy efficiency of the system, neglected in previous works, is a relevant purpose of an energy apportionment policy.

(2) Recognise 8 different energy apportionment policies already proposed in different fields to provide a common understanding of the energy apportionment problem. In addition, our work formalises 3 of them not previously presented mathematically.

(3) Propose 2 alternative novel policies based on cooperative game theory not previously used in energy apportionment. These policies provide a strong fairness notion and a rich incentive structure.

(4) Analyse the information requirement and computational complexity of the 10 energy apportionment policies.

(5) Evaluate the fairness of the 10 policies employing the identified 5 fairness properties.

Our analysis shows that there is a trade-off between fairness and the other evaluation criteria, and provides guidelines to select an policy depending on the purpose of the energy apportionment and the characteristics of the system. In particular, for the 5 formal fairness properties we show that the proposed game theoretical policies provide the strongest fairness. However, these policies have a high information requirement and computational complexity, and thus other simpler policies are identified to provide a satisfactory compromise when the fairest policies become unpractical. We also classify the policies based on the type of input information used, and pinpoint that if surrogate information (e.g., usage time) is used in the apportionment, this has to correlate with the energy consumption of the system in order to provide correct incentives to reduce the energy consumption. 
Note that apportionment might be a basis of setting prices or further developing a charging scheme. However, pricing schemes are not in the scope of this paper.

The rest of the paper is structured as follows: Section 2 introduces our system model as well as the background, and section 3 describes the 10 energy apportionment policies in their application context. Section 4 compares the policies in terms of required information and computational complexity. In section 5 we leverage game theory to provide a formal study of the fairness characteristics of the different energy apportionment policies. Section 6 summarises and discusses the insights of our analysis, and section 7 describes the relevant work in the area of energy accounting as well as an overview of energy management. Finally, section 8 concludes our work and provides future directions.

\section{NOTATION AND BACKGROUND}

In order to formulate the different energy apportionment policies we first introduce our system model, a time model employed to formalise some policies and the corresponding notation used in the rest of the paper. We also introduce the apportionment policy definition and the required background from game theory to understand our analysis.

\subsection{System model}

An energy consuming system is composed by the set of entities $N=\{1,2, \ldots, n\}$ that cause the system which they are part of to consume energy. We distinguish two types of system interpretations: (1) common resource, where the entities share a common energy resource, and (2) individual resource, where each entity has an energy resource. Fig. 2 shows an illustration of the two types of systems.
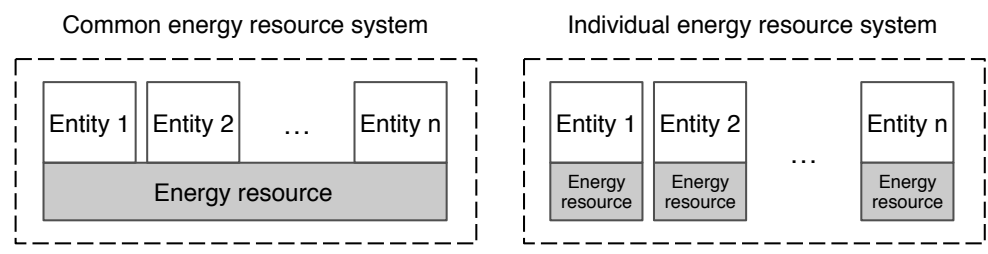

Fig. 2: Illustration of the system models.

Examples of a common resource system are the computers in a datacenter or a battery-powered device shared by different applications, whereas a set of mobile devices or sensor nodes in an ad-hoc or sensor network are systems with individual resources.

2.1.1 Common resource system. $E(N)$ is the total energy consumption for a system configuration of the $N$ entities sharing a common resource. We assume that equipment to measure $E(N)$ is available, e.g., measuring the power consumption of a phone by intercepting the battery terminals [Vergara et al. 2014b] or the mains connected to a home appliance [Cheng et al. 2012].

$E(S)$ is the isolated energy consumption of the entities in $S \subset N$. Thus, $E(\{i\})$ is the isolated energy consumption of the single entity $i \in N$. E $(\{i\})$ represents the energy consumption of the entity $i$ in the hypothetical case that it was alone in the system, not considering the interaction of the other entities in the system. This quantity is a construct which is estimated (generally not measurable), for example a single application running alone on a mobile device or computer. 
2.1.2 Individual resource system. $E_{i}(N)$ is the individual energy consumption of the entity $i \in N$ given a system configuration of entities $N$. We assume that equipment to measure $E_{i}(N)$ at each entity $i$ is available. The total system consumption is $\sum_{i \in N} E_{i}(N)$, e.g., the total consumption of a network of sensor nodes is the sum of nodes' consumption in a network configuration ${ }^{1}$.

$E_{i}(S): i \in S, S \subset N$, is the individual energy consumption of the entity $i$ given a system configuration of entities $S$. This is a construct which is estimated (generally not measurable). The total energy consumption for a given $S \subset N$ is $\sum_{i \in S} E_{i}(S)$.

2.1.3 Discrete-event time model. The formalisation of some policies require to model time and thus we employ a discrete-event formal framework introduced in the context of real-time systems [Lee 1999]. This section provides an overview of the model.

Atomic events occur along a physical time line where every event $e=(t, v)$ is defined by a time point $t \in \mathbb{R}$ and a value $v \in V$. The value $v$ is a flexible representation specific to the system.

A discrete-event signal $s$ is a set of events and it cannot have two identical events. $D$ is the set of all all signals $(s \in D)$. The definition of a functional signal [Lee 1999] states that the events in any $s$ can be enumerated chronologically, there is a single event per $t$ and that between any two time points there is a finite number of events.

Tuples of signals are used for naming purposes. A tuple $\mathbf{s}$ of $N$ signals is defined as $\mathbf{s}=\left[s_{1}, \ldots, s_{N}\right]$ where the set of all such tuples is $D^{N}$.

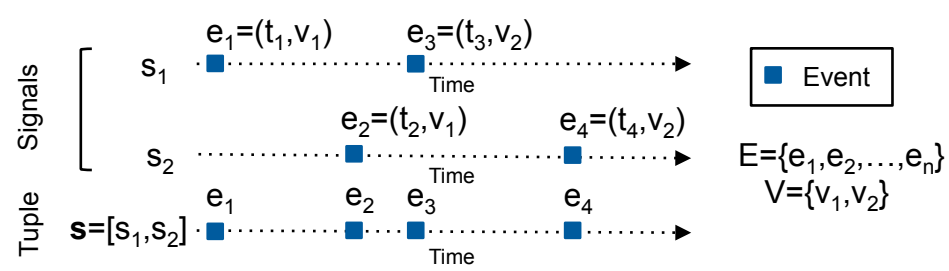

Fig. 3: Example of the time model for two discrete signals and a tuple.

Fig. 3 shows an example of two discrete signals $s_{1}$ and $s_{2}$ with two value types $\left(v_{1}\right.$ and $\left.v_{2}\right)$ which are part of a tuple $\mathbf{s}$.

\subsection{Energy apportionment policy}

An energy apportionment policy $\Pi$ prescribes the share $\pi_{i} \in \mathbb{R}$ to an entity $i \in N$ of the total system consumption of the system composed by $N$ entities. A policy may use different input information to apportion the total energy cost among the entities. Equation 1 and 2 define $\Pi$ for a common and individual resource system respectively:

$$
\begin{gathered}
\Pi(E(N), N, \ldots)=\left(\pi_{1}, \pi_{2}, \ldots, \pi_{n}\right) \text { in } R^{n} \\
\Pi\left(E_{1}(N), \ldots, E_{n}(N), N, \ldots\right)=\left(\pi_{1}, \pi_{2}, \ldots, \pi_{n}\right) \text { in } R^{n}
\end{gathered}
$$

An apportionment policy should completely apportion the total energy cost: $\sum_{i \in N} \pi_{i}=E(N)$ and $\sum_{i \in N} \pi_{i}=\sum_{i \in N} E_{i}(N)$ for common and individual resource respectively. This is referred to as efficiency in the next section.

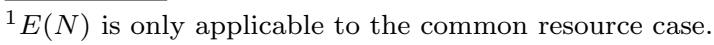




\subsection{Game theory for energy apportionment}

Cooperative game theory has been extensively employed as a method to model and solve cost division problems. We show that it can be employed for energy apportionment to determine the energy contribution of the entities in the system.

A transferable utility game (TU-game) in coalitional form is defined by a set of players $N$ (the entities) and a characteristic function $c$ that models cost. A coalition is a subset $S \subseteq N$, where $N$ is the grand coalition and $\emptyset$ is the empty coalition. The characteristic function assigns a cost to each coalition $S, c: 2^{N} \rightarrow \mathbb{R}$, where $c(\emptyset)=0$ by convention. We employ the system energy function $E$ as the characteristic function $c$ to model the energy consumption of the system. A $n$-player game is defined by the pair $(N, E)$, where the players will divide $E(N)$ given the power structure in the grand coalition expressed by the characteristic function.

Properties of characteristic functions and their structure classify the games providing insights about the usage of apportionment policies. A cooperative game is monotone if $E\left(S_{1}\right) \leq E\left(S_{2}\right)$ when $S_{1} \subseteq S_{2} \subseteq N$. A game is subadditive if $E\left(S_{1}\right)+E\left(S_{2}\right) \geq E\left(S_{1} \cup S_{2}\right)$ given $S_{1}, S_{2} \subseteq N$ and $S_{1} \cap S_{2}=\emptyset$, which means that the coalitions have an incentive to cooperate since they lower the costs together. An essential game is characterised by $E(N)<\sum_{i \in N} E(\{i\})$. Finally, a game is concave if $E\left(S_{1} \cup\{i\}\right)-E\left(S_{1}\right) \geq E\left(S_{2} \cup\{i\}\right)-E\left(S_{2}\right)$ for $i \in N, S_{1} \subset S_{2} \subseteq N \backslash\{i\}$. This implies that the incentive for joining a coalition increases when the coalition grows.

Given all possible divisions of the total cost, a single point solution concept for a cooperative game $(N, E)$ provides a unique vector representing the cost division (i.e., apportionment) to each player based on a given criteria. Solution concepts developed for cooperative TU-games can be employed as apportionment policies and its resulting $\pi$ can fulfil certain properties. Efficiency states that the total cost is completely apportioned: $\sum_{i \in N} \pi_{i}=E(N)$. We consider efficiency a minimum requirement for an apportionment policy, and thus, for our analysis all the policies satisfy the efficiency property.

Individual rationality, $\pi_{i} \leq E(\{i\}), \forall i \in N$, implies that a single player has lower or equal cost while cooperating than being alone. An apportionment is an imputation if it satisfies efficiency and individual rationality. We refer to the set of apportionments which satisfy individual rationality and efficiency for a given $E$ as the imputation set $I(E)$.

The game and apportionment properties will be used to compare different energy apportionment policies.

\section{ENERGY APPORTIONMENT POLICIES}

We first categorise energy apportionment policies based on the type of input information. An energy-based policy (EB) apportions the system energy using different constructs with energy as the only type of input information. A surrogate-based policy (SB) may employ any type of information except energy. A hybrid policy would combine energy and surrogates.

This section presents 10 different policies. We survey 5 of the most used energy-based apportionment policies, propose 2 new policies originated from cooperative game theory and formalise 3 existing surrogatebased policies not described mathematically in earlier work. We briefly explain each policy and provide a brief description of application examples. The application of the policies and their different contexts are explained in section 7 (related works). Table I summarises the 10 policies and describes in brief their intuition.

\subsection{Energy-based policies}

This section describes 7 energy-based policies. All policies can be formulated for common and individual resource systems, but due to space limitation and to simplify the presentation we present them using the common resource notation.

Policy 1: Equal division: The total energy consumption is divided equally by the number of entities: 
Table I. : Summary of the 10 energy apportionment policies and their intuition.

\begin{tabular}{|c|c|c|c|}
\hline Nr. & Name & Type & Description \\
\hline 1 & Equal division & EB & Apportion the total energy consumption equally to all entities. \\
\hline 2 & Proportional to isolation & EB & Apportion proportionally to each entity's isolated energy. \\
\hline 3 & Marginal contribution & EB & Apportion proportionally to each entity's marginal contribution. \\
\hline 4 & Isolation energy and remainder & EB & Isolated consumption and apportion the rest equally. \\
\hline 5 & Shapley value & EB & $\begin{array}{l}\text { Apportion the average marginal contribution with respect to all } \\
\text { permutations on the set of entities. }\end{array}$ \\
\hline 6 & Nucleolus & EB & Minimise the unhappiness of the most unhappy entity. \\
\hline 7 & Least square nucleolus & EB & Minimise the variance of all entities' unhappiness. \\
\hline 8 & Proportional to activity & SB & $\begin{array}{l}\text { Apportion proportionally to each entity's activity, using some } \\
\text { notion of activity (surrogate). }\end{array}$ \\
\hline 9 & Time slicing & SB & $\begin{array}{l}\text { For each time interval apportion equally among the active entities. } \\
\text { If no entity is active apportion equally among all entities. }\end{array}$ \\
\hline 10 & Last active & SB & $\begin{array}{l}\text { For each time interval apportion equally among the active entities. } \\
\text { If no entity is active apportion to the last active entity. }\end{array}$ \\
\hline
\end{tabular}

Isolated energy consumption: Energy consumption of an entity if this was alone in the system (see section 2.1.1).

Marginal contribution of an entity: Energy when an entity is running in the system minus when it is not running.

EB: Energy-based apportionment policy. SB: Surrogate-based apportionment policy.

$$
\pi_{i}=\frac{E(N)}{|N|}
$$

This apportionment policy is regarded as the simplest policy and has been proposed in the context of energy-efficient buildings [Hay and Rice 2009] as well as in energy accounting for wireless sensor networks [Fonseca et al. 2008].

\section{Policy 2: Proportional to isolation:}

The resulting apportionment is proportional to the isolated energy consumption of each entity. The policy is the same as the pro rata division of $E(N)$ considering the isolated or stand-alone energy:

$$
\pi_{i}=\frac{E(\{i\})}{\sum_{j \in N} E(\{j\})} E(N)
$$

The energy consumption of a multicore CPU is shared by the different entities in proportion to their energy usage in isolation (in a single core) [Ryffel et al. 2009]. The same idea has been also proposed to share the cost of heating, ventilation, and air conditioning (HVAC) systems [Tsao et al. 2014].

The most basic version of this policy is proposed for mobile application energy profiling, where the contribution of an application is assumed to be its consumption in isolation $\pi_{i}=E(\{i\})$ [Mittal et al. 2012; Neugebauer and McAuley 2001; Vergara et al. 2014b]. However, the basic version does not satisfy efficiency since the total energy consumption is not always the sum of the isolated energy consumption of the entities, and thus we only consider the normalised version of the policy.

\section{Policy 3: Marginal contribution:}

This policy considers that the contribution of an entity to the total consumption is its marginal contribution $E(N)-E(N \backslash\{i\})$, i.e., the total consumption when the entity is running minus the consumption when the entity is not running. The policy is normalised using all the marginal contributions:

$$
\pi_{i}=\frac{E(N)-E(N \backslash\{i\})}{\sum_{j \in N}[E(N)-E(N \backslash\{j\})]} E(N)
$$


This policy was proposed for operating systems to account for runtime energy consumption of individual system hardware and software entities in multicore systems [Ryffel et al. 2009; Ryffel 2009].

Similarly to Policy 2, a basic version of this policy was proposed in the area of graphical user interfaces [Zhong and Jha 2003], where an entity is prescribed its marginal contribution $\pi_{i}=E(N)-E(N \backslash\{i\})$. This basic version does not satisfy efficiency since the sum of marginal contributions is not necessarily equal to the total energy consumption.

Policy 4: Isolation energy and remainder: The policy prescribes the allocation of costs as a function of the energy consumption in isolation plus proportionally dividing the additional cost/benefit of non-isolated use:

$$
\pi_{i}=E(\{i\})+\frac{E(N)-\sum_{j \in N} E(\{j\})}{|N|}
$$

The usage of this policy is originated in the energy-efficient building community [Hay and Rice 2009], where the personal consumption is estimated first and the rest is divided evenly (e.g., base energy load of building elements such as HVAC). Similarly, in the context of wireless sensor networks, the energy cost of messages received by a certain task is prescribed to the task and the rest (e.g., synchronisation or message loss) is shared evenly among the tasks [Kellner 2010].

Policy 5: Shapley value: Given the marginal contribution of $i$ to a coalition $S \subseteq N \backslash\{i\}$ given by $E(S \cup\{i\})-E(S)$, the Shapley value computes the average marginal contribution of $i$ averaging over all the possible sequences through which the grand coalition can be built from the empty coalition [Shapley 1953]:

$$
\pi_{i}=\sum_{S \subseteq N \backslash\{i\}} \frac{|S| !(|N|-|S|-1) !}{|N| !}[E(S \cup\{i\})-E(S)]
$$

The Shapley value is a well known single value solution from game theory applied to many cost sharing problems in computer science [Misra et al. 2010; Sereno 2012] as well as in other fields [Fiestras-Janeiro et al. 2011]. Recently it has been argued to be the ground truth for energy accounting in mobile devices [Dong et al. 2014].

Policy 6: Nucleolus: Given an imputation $\pi=\left(\pi_{1}, \pi_{2}, \ldots, \pi_{n}\right)$, i.e., an efficient apportionment satisfying individual rationality, the excess $e(S, \pi)=E(S)-\sum_{i \in S} \pi_{i}$ measures the satisfaction of a coalition from apportionment $\pi$.

Let $\theta(\pi)$ denote a vector that contains the excess for each coalition $S \subseteq N$ in non-decreasing order given an imputation $\pi$. The nucleolus is the apportionment $\pi$ which maximises $\theta(\pi)$ over all the other imputations $\pi^{*}$ in the imputation set $I(E)$ using the lexicographic ordering $\left(\geq_{L}\right)$ [Schmeidler 1969]:

$$
\pi \mid \theta(\pi) \geq_{L} \theta\left(\pi^{*}\right) \text { for all } \pi, \pi^{*} \in I(E)
$$

The nucleolus is commonly computed by solving a sequence of linear programming (LP) problems [Maschler et al. 1979]. The prenucleolus computes an apportionment following the same method for the set of all efficient apportionments, providing an apportionment when the imputation set is empty.

Policy 6 is proposed in this paper for energy apportionment due to its interesting properties (section 5), and to the best of our knowledge it has not been proposed as an energy apportionment policy.

Policy 7: Least square nucleolus: For a given imputation $\pi$ the average excess $\bar{e}$ is:

$$
\bar{e}(\pi)=\frac{1}{2^{|N|}-1} \sum_{S \subset N} e(S, \pi)
$$

Authors' preprint 
The least square nucleolus (LSN) is the imputation that minimises $\sum_{S \subset N}(e(S, \pi)-\bar{e}(\pi))^{2}$, i.e., an imputation whose associated excesses are closest to the average excess under the least square criterion [Ruiz et al. 1996]. The least square prenucleolus (LSP) is the efficient apportionment that follows the same method.

The computation of the LSN is performed in two steps: (1) LSP $\pi^{P}$ is calculated using Equation 9,

$$
\pi_{i}^{P}=\frac{E(N)}{|N|}+\frac{1}{|N| 2^{|N|-2}}\left[|N| \sum_{S: i \in S} E(S)-\sum_{S \subset N}|S| E(S)\right]
$$

followed by (2) a recursive algorithm that computes the LSN $(\pi)$ using the LSP apportionment $\pi^{P}$ as input. For each step in the algorithm a 0 is given to the entities with a negative excess in some of the earlier steps, dividing the aggregated negative excess from the previous step evenly among rest of the entities. The algorithm stops when no entity gets a negative excess, which results in an apportionment $\pi$ which is an imputation.

To the best of our knowledge LSN has not been proposed for energy apportionment policies.

\subsection{Surrogate-based policies}

This section employes the time model described in section 2.1.3 to formalise 3 previously proposed surrogatebased policies. These policies are selected because we believe that they are good representatives of surrogatebased policies.

Policy 8: Proportional to activity: Given two events that signify that an entity becomes active and inactive respectively $(V=\{0,1\}$, where $v=1$ for active and $v=0$ for inactive), the policy apportions energy proportionally to the activity time. Let $s_{i}$ be the signal of events of entity $i$, the apportionment policy is defined as:

$$
\pi_{i}=\frac{a\left(s_{i}\right)}{\sum_{j \in N} a\left(s_{j}\right)} E(N)
$$

where $a(s): S \rightarrow \mathbb{R}$ is a function that provides the total active time of entity $i$ by performing a sum of the periods between events with values 1 and 0 . This policy has been proposed to model the occupancy of a building to divide its energy consumption among the occupants [Hay and Rice 2009].

Policy 9: Time slicing: This policy considers that multiple entities may be active simultaneously and there exist idle time. Given the sequence of events $\left(e_{1}, e_{2}, \ldots, e_{m}\right)$ temporally sorted in $\mathbf{s}$, a sequence of intervals $J$ is computed. An interval $j \in J$ is given by the delimiting events' time points $\left[t_{j}, t_{j+1}\right)$, where $e_{j}=\left(t_{j}, v\right)$, $e_{j+1}=\left(t_{j+1}, v^{\prime}\right)$ and $t_{j}<t_{j+1} . T$ is the period between the first and last event.

The Boolean function $a(i, j): N \times J \rightarrow\{0,1\}$ denotes whether an entity $i$ is active during interval $j$ or not. Function $f(i, j): N \times J \rightarrow[0,1]$ divides an interval among the active entities proportionally, or evenly if the entities are inactive.

$$
f(i, j)= \begin{cases}\frac{1}{|N|} & \text { if } \sum_{i \in N} a(i, j)=0 \\ \frac{a(i, j)}{\sum_{i \in N} a(i, j)} & \text { otherwise }\end{cases}
$$

This function is used to define a policy as described in Equation 11:

$$
\pi_{i}=\frac{1}{T} \sum_{j \in J}\left[f(i, j)\left(t_{j+1}-t_{j}\right)\right] E(N)
$$


The policy is used to apportion a micro controller's consumption in a scenario of a distributed database over a network of sensors [Kellner 2010] and a building's energy to tenants [Thakur et al. 2014]. Also, this policy is used for apportionment of a single core processor [Flinn and Satyanarayanan 1999b].

Policy 10: Last active: Given the intervals $J$, the energy consumption for each interval is apportioned among the active entities. During any interval that all entities are inactive, the energy consumed during the interval is assigned to the last active entity.

For each interval $j$ the energy consumption is denoted by $E(N, j)$, where $\sum_{j \in J} E(N, j)=E(N)$. The Boolean function $l(i, j): N \times J \rightarrow\{0,1\}$ denotes if entity $i$ is the last active entity of interval $j$ by simply keeping track of the latest "become active" event $(v=0)$.

$$
g(i, j)= \begin{cases}l(i, j) & \text { if } \sum_{i \in N} a(i, j)=0 \\ \frac{a(i, j)}{\sum_{i \in N} a(i, j)} & \text { otherwise }\end{cases}
$$

Policy 10 is defined using function $g(i, j)$ :

$$
\pi_{i}=\sum_{j \in J} g(i, j) E(N, j)
$$

The policy provides incentives to aggregate the active events of the different entities. This policy is used to apportion the energy tails created by inactivity timeouts for different smartphone components [Pathak et al. 2012].

The following sections compare the presented 10 energy apportionment policies based on 3 criteria: input information, computational complexity and fairness. Section 4 analyses the input information and computational complexity, whereas in section 5 we analyse the fairness of the different policies employing five formal criteria.

\section{INPUT INFORMATION AND COMPUTATIONAL COMPLEXITY}

The presented policies require different amounts of input information to perform the apportionment and obtaining this information might be harder depending on the domain of the input and the system. In addition, the amount of input information has an impact on the computational complexity of the policies. We analyse and compare the computational complexity of the policies and provide pointers to efficient solutions in the literature for the most complex policies.

Table II summarises the comparison of the policies using the different criteria and we describe the details of the comparisons next.

\subsection{Required input information}

Section 4.1.2 discusses the amount of information needed for each policy, whereas section 4.1.1 compares the domain of the input information, i.e., surrogate-based and energy-based policies.

4.1.1 Information type. We categorised the policies based on input type: energy consumption or surrogates. Dividing energy consumption based on energy information appears as a natural choice: if the cost function is known, it is natural to use it to apportion the cost. However, while obtaining $E(N)$ is straightforward, measuring or estimating $E(S) \mid S \subset N$ is complex (if not impossible) given the shared nature and lack of visibility of energy consumption in current systems. We observe that obtaining $E(N \backslash\{i\})$ seems qualitatively harder than $E(\{i\})$ since the first already considers the energy resulting from the use of a shared resource, whereas the second one considers the energy in isolation without any interaction with the other 


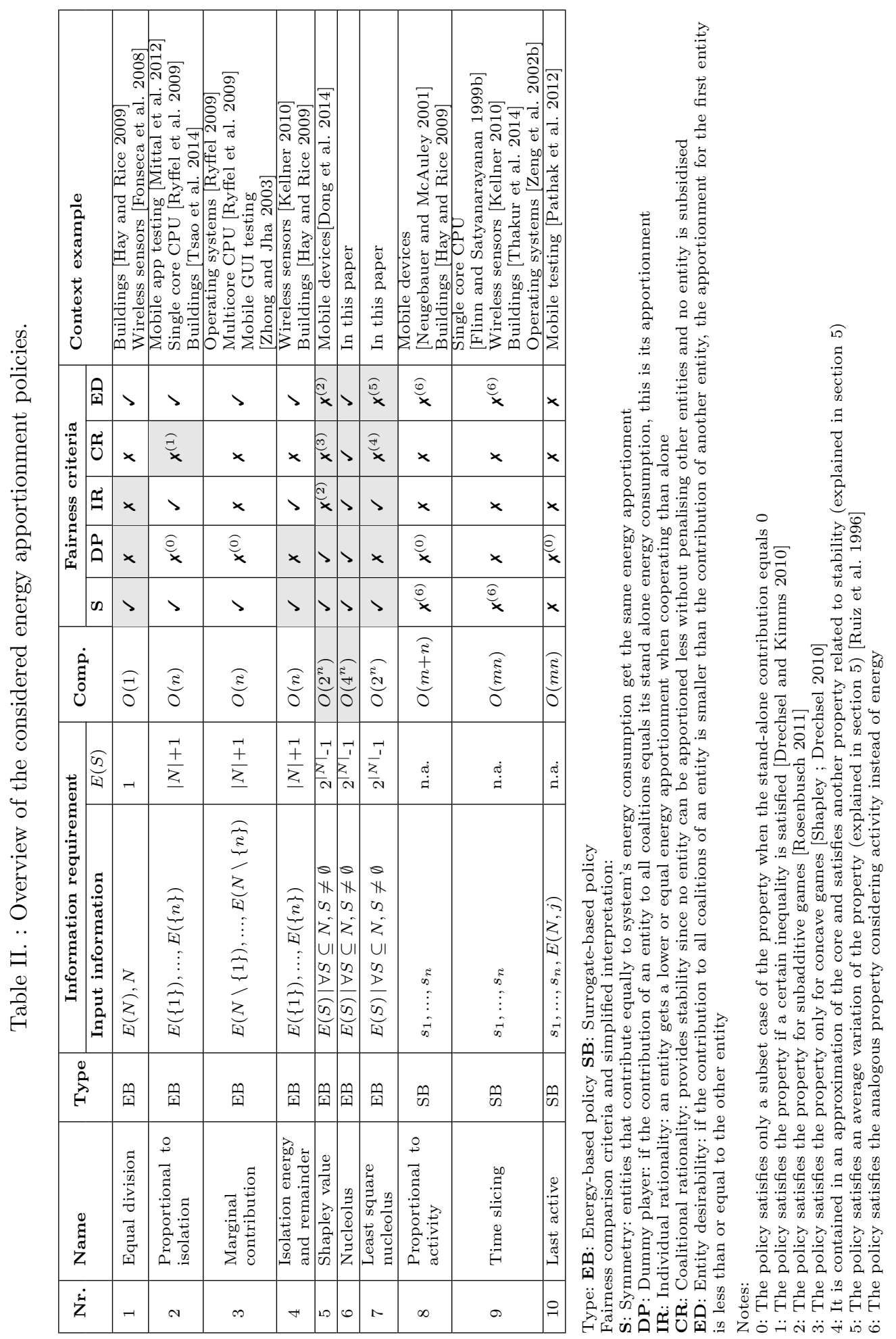


entities. Approximations of $E(S)$ range from accurate energy models [Vergara et al. 2014b; Pathak et al. 2012; Mittal et al. 2012] to measuring some of the observable $S \subset N$ and approximating the unobserved values using regression techniques [Dong et al. 2014].

Surrogate-based policies perform energy apportionment by translating the problem to another domain outside energy consumption. This can simplify the apportionment problem and the data acquisition (e.g., surrogate information is easily accessible using performance counters in computers [Bertran et al. 2010] or external activity sensors in buildings [Saha et al. 2014; Cheng et al. 2012]). However, the surrogate input does not necessarily reflect how energy is consumed in the system. For example, the amount of data sent does not reflect the energy consumed in the wireless interface but it is easily accessible [Vergara et al. 2014b].

4.1.2 Information quantity. Columns 4 and 5 in Table II show the amount of needed information for each policy. Policy 1 employes the minimum input quantity: $E(N)$ and $N$. Policies 2, 3 and 4 require the energy consumption of $|N|$ subsets. Policies 2 and 4 consider the energy in isolation $E(\{i\})$ and 3 considers the system energy excluding a single entity $E(N \backslash\{i\})$. Policies 5,6 and 7 present the highest information requirement, requiring the energy consumption of all the subsets of $N\left(2^{|N|}\right)$ to build the game.

Apart from $E(N)$, the input the surrogate-based policies is from another domain than energy consumption. Policies 8-10 require at least a signal $s_{i}$ per entity ( $n$ signals) with the events collected from the system. Additionally, Policy 10 requires $E(N)$ per interval.

Thus, depending on the available information for a system some policies seem more appealing than others. It is worth mentioning that there exist ways to approximate games with incomplete information (e.g., missing information about the consumption of some subsets) [Polkowski and Araszkiewicz 2003; Masuya and Inuiguchi 2009].

\subsection{Computational complexity}

This section presents the computational complexity of the policies. We analyse the general complexity and provide pointers for problem specific complexity reduction for the most complex policies. Column 6 in Table II shows the complexity of each policy. The size of the input (information quantity) is a highly influential aspect in the computational complexity of the policies.

4.2.1 General complexity. Policies 1 to 4 show low complexity. Policy 1 performs a single operation and its complexity is $O(1)$. Policies 2, 3 and 4 are computed in $O(n)$ given the sum of $|N|$ elements. The computational complexity of Policy 5 increases exponentially with the number of entities $O\left(2^{n}\right)$. Policy 6 is the most complex policy, computed by solving a sequence of at most $|N|$ number of linear programming (LP) problems of decreasing dimension [Maschler et al. 1979] or a single large-scale LP problem with $O\left(4^{n}\right)$ constraints [Puerto and Perea 2013]. The complexity of Policy 7 is $O\left(2^{n}\right)$ due to two steps: (1) the calculation of LSP using Equation 9 involves iterating over the $2^{n}$ coalitions, and (2) a maximum of $|N|$ operations are performed by the algorithm to compute LSN.

The complexity of Policies 8-10 depends on the number of events $m$ of all the entities and the functions employed. The complexity of Policy 8 is $O(m+n)$ due to analysing $m$ events when using function $a(s)$ and the sum of $|N|$ entities. Policy 9 and 10 employ $m-1$ intervals and for each interval and entity, and the policies compute the apportionment using functions $f(i, j)$ and $g(i, j)$ respectively, leading to a $O(m n)$ complexity.

4.2.2 Optimisations. There exist approximation techniques for the Shapley value (Policy 5) where the computational complexity increases linearly with the number of players [Fatima et al. 2008; Castro et al. 2009]. There are also exact solutions that take advantage of some particular game representation to reduce the complexity (e.g., $O(n)$ and $O\left(n^{2}\right)$ for a tree [Megiddo 1978] and weighted graph representation respectively [Deng and Papadimitriou 1994]). 
Efficient solutions have been found for the nucleolus (Policy 6) for specific game structures, such as a tree structure where it is computed in $O\left(n^{3}\right)$ [Megiddo 1978] or a weighted graph in $O\left(n^{2}\right)$ [Deng and Papadimitriou 1994]. However, NP-hardness has been shown for other specific game representations (e.g., minimum spanning tree game [Faigle et al. 1998]). Reducing the computation requirement for the nucleolus is an ongoing work.

To sum up, the computational complexity is important to consider when performing energy apportionment of systems, especially with a large number of entities. Policies 5-7 exhibit higher complexity than the rest of policies.

\section{FAIRNESS IN ENERGY APPORTIONMENT}

Except Policy 5, the application of all the presented policies to apportion energy consumption has been promoted as fair by the authors using them (see Table II) with no formal justification. We analyse fairness by first introducing a formal notion of fairness that leverages game theory in section 5.1. Section 5.2 describes in detail the fairness properties satisfied by each policy, which is summarised in Table II.

\subsection{Fairness criteria}

In this section we propose that fairness can be characterised by selecting five different criteria. These criteria capture properties that are important when apportioning energy consumption in an intuitive and rational manner. We introduce and motivate each criteria for energy apportionment:

Symmetry: if $E(S \cup\{i\})=E(S \cup\{j\})$ for all $S \subseteq N \backslash\{i, j\}$, then $\pi_{i}=\pi_{j}$. If the marginal contribution of $i$ and $j$ to all coalitions is the same, their apportionment is the same. Symmetry is a desirable property for an apportionment policy since two entities that contribute equally to the energy consumption of the system should intuitively receive the same apportionment.

Dummy player: if $E(S \cup\{i\})-E(S)=E(\{i\})$ for all $S \subseteq N \backslash\{i\}$, then $\pi_{i}=E(\{i\})$. If the marginal contribution of entity $i$ to all the coalitions is the same as its stand-alone value, then the latter is her value.

The property reflects the fact that the consumption of an entity in the system can be independent of the other entities, and thus it is intuitive to assume that if the entity does not impact the energy consumption of the system or the rest of the entities when sharing the resource, this entity should be prescribed its stand-alone cost. The property also includes the case of when $E(\{i\})=0$, which should intuitively lead to a prescription of no cost. This basic case of dummy player is also known as null player.

Individual rationality: This property was briefly introduced in section 2.3. We recall that an apportionment is individually rational if $\pi_{i} \leq E(\{i\}), \forall i \in N$. An apportionment policy satisfies individual rationality if it results in such an apportionment when it exists. Recall that when such an apportionment exists it is called an imputation, and the set of such imputations is denoted $I(E)$. It is well known that $\sum_{i \in N} E(\{i\}) \geq E(N)$ iff $I(E) \neq \emptyset$. Hence, the property is formally defined as: if $I(E) \neq \emptyset$ then $\pi_{i} \leq E(\{i\}), \forall i \in N$.

We argue that individual rationality is an appropriate fairness criteria since: (1) When all the entities contribute to lower the total energy consumption compared to the sum of entities' stand-alone consumption, it is unintuitive to prescribe an entity more that it would consume alone, and (2) individual rationality provides incentives to cooperate since every entity's apportionment in the system is lower than what its energy consumption would have been in isolation.

Coalitional rationality: An apportionment is coalitionally rational if $\sum_{i \in S} \pi_{i} \leq E(S) \forall S \subseteq N$. An apportionment policy satisfies coalitional rationality if it results in such an apportionment when it exists. 
In game theory, the set of apportionments that satisfy coalitional rationality is referred to as the core $e^{1}$ of a game denoted by $C(E)$. Then, coalition rationality is formally defined as:

if $C(E) \neq \emptyset$ then $\sum_{i \in S} \pi_{i} \leq E(S) \forall S \subseteq N$.

This property is relevant since it implies that no entity or coalition is subsidised: if one entity would be prescribed less cost in a coalitionally rational apportionment, another entity would be penalised, i.e., the apportionment is Pareto optimal [Drechsel 2010]. Additionally, this property is attractive for energy apportionment in cooperative systems, since selecting a coalitionally rational apportionment leads to the case where no coalition could reach a better result than in the grand coalition. This introduces a stability notion by avoiding any incentive to divide the grand coalition.

Entity desirability: For the final criterion we adapt the player desirability criterion introduced by Rosenbusch [Rosenbusch 2011]. Entity $i$ is said to be more desirable than entity $j(i \succeq j)$ if the energy consumption contribution of $i$ to all coalitions that exclude both entities is less than or equal to the one that $j$ would contribute: $E(S \cup\{i\}) \leq E(S \cup\{j\}) \forall S \subseteq N \backslash\{i, j\}$. The criterion states that a more desirable entity gets an apportionment less than or equal to the apportionment of the less desirable entity: $i \succeq j \Longrightarrow \pi_{i} \leq \pi_{j}$.

We refer to $i$ and $j$ as comparable if $i \succeq j$ or $j \succeq i$. We believe that the criterion is intuitive, since a lower contribution of an entity than another should be reflected in the apportionment.

\subsection{Fairness comparison}

The fairness criteria column in Table II shows whether a policy always satisfies the symmetry, dummy player, individual rationality, coalitional rationality and entity desirability properties. The 21 shaded cells in the table out of the 50 relate to known results based on previous extensive analysis from game theory literature. Table III in the appendix provides the references to the existing results tracing each property to each policy.

The other 29 outcomes are our contributions. These results strictly follow from the respective fairness property and policy definitions. The detailed analysis of the policies in this section is structured per property, where we provide the intuition of why a certain policy satisfies or does not satisfy each property. We refer the interested reader to the electronic appendix (attached at the end of this manuscript) where all the proofs can be found. We also refer to each of our proofs in the text.

5.2.1 Symmetry. All policies satisfy symmetry except for the surrogate-based policies (Policies 8, 9 and 10). The proofs for the symmetry property can be found in section A of the appendix.

Since the Equations 3-9 of Policies 1 to 7 do not consider any information other than the value of the system energy function $E$, two entities contributing with the same energy to all coalitions always get the same apportionment. For example, Policy 1 (Equal division) trivially satisfies symmetry since it always apportions the same value to all entities, or Policy 2 (Proportional to isolation) divides the stand-alone energy by the same value for all entities (Theorem A.1). Similarly, Policy 3 (Marginal contribution) satisfies symmetry since the denominator is equal for all entities (Theorem A.2).

Policies 8, 9 and 10 (surrogate-based policies) do not satisfy symmetry since activity and energy are not necessarily correlated. Thus, even if two entities are symmetric in terms of energy, this is not reflected in the apportionment policies 8, 9 and 10 which consider activity as input (Theorems A.3, A.4 and A.5 respectively).

5.2.2 Dummy player. Policies 5 and 6 (Shapley value and Nucleolus), are the only policies satisfying the dummy player criterion. The general reason for not satisfying the property is that the policies perform a certain operation that prevents an entity from getting its isolated energy consumption. The proofs for the dummy player property can be found on section B of the appendix.

\footnotetext{
${ }^{1}$ Even though the core presents desired properties, we do not consider the core as an apportionment policy and use it as a comparison criterion since the core is rarely unique and may be empty.
}

Authors' preprint 
The normalisation factor of Equations 4 and 5 is the main reason for not satisfying dummy player for Policy 2 (Proportional to isolation) and Policy 3 (Marginal contribution) (Theorems B.1 and B.2 respectively). Even if an entity contributes with its isolated energy consumption $E(\{i\})$ to all coalitions, Policy 2 and 3 normalise the result and thus the apportionment does not always result in that isolated energy consumption.

Surrogate-based policies $(8,9$ and 10) do not always satisfy the criterion even in the case where the activity function is equal to the system energy function. Policy 8 (Proportional to activity) employes a normalisation factor (similarly to Policy 2) (Theorem B.3). Policy 9 (Time slicing) divides the idle energy equally among all entities (Theorem B.4). Policy 10, Last active, prescribes a greater energy consumption to the last active entity (Theorem B.5).

Finally, policies $2,3,8$ and 10 satisfy the special case when the contribution of an entity is 0 to all coalitions. For surrogate-based policies, it is intuitive that if an entity does not have a surrogate attribute in a scenario (e.g., it does not have any activity), it does not consume energy in isolation and vice versa. Hence, $\mathrm{a}(\mathrm{i})=0$ iff $\mathrm{E}(\{\mathrm{i}\})=0$.

It is easy to verify this so called null player property for Policy 2 (Proportional to isolation), Policy 3 (Marginal contribution) and Policy 8 (Proportional to activity) by looking at Equations 4, 5 and 10: if the contribution of an entity is 0 , the entity is prescribed no energy since $E(\{i\}), a(i)$ and $E(N)-E(N \backslash\{i\})$ become 0 respectively, and thus $\pi_{i}=0$ (Theorems B.6, B.7 and B.8 respectively). For Policy 10 (Last active) if an entity is never active it cannot be the last active entity and thus it is prescribed no energy (Theorem B.10).

5.2.3 Individual rationality. Policy 2 (Proportional to isolation), Policy 4 (Isolation energy and remainder), Policy 6 (Nucleolus) and Policy 7 (Least square nucleolus) always result in an individually rational apportionment when it exists. The proofs for the individual rationality property can be found on section $\mathrm{C}$ of the appendix.

Policies 2 and 4 (Proportional to isolation and Isolation energy and remainer), always satisfy individual rationality since the apportionment is performed by considering the isolated energy consumption $E(\{i\})$ (Theorems C.1 and C.3).

However, Policy 3 (Marginal contribution) does not always satisfy individual rationality mainly due to Equation 5 does not consider the energy consumption of $E(\{i\})$ (Theorem C.2). Policy 5 (Shapley value) does not always satisfy this property, but if the resulting game based on the energy consumption values $E(S)$ is subadditive Policy 5 will satisfy individual rationality.

Similarly, the surrogate-based policies (policies 8, 9 and 10) do not satisfy the property since the activity of an entity is not necessarily correlated with energy consumption (Theorems C.4, C.5 and C.6).

5.2.4 Coalitional rationality. Policy 6 (Nucleolus) is the only apportionment policy that satisfies coalitional rationality when such a solution exists. Coalitional rationality implies individual rationality when $|S|=1$, and thus Policies $1,3,8,9$ and 10 that do not always satisfy individual rationality do not satisfy coalitional rationality either.

Policy 2 (Proportional to isolation) does not always satisfy coalitional rationality, but it will satisfy the criterion if the following restrictive inequality holds [Drechsel and Kimms 2010]:

$$
\frac{\sum_{i \in S} E(\{i\})}{\sum_{i \in N} E(\{i\})} \leq \frac{E(S)}{E(N)} \text { for all } S \subseteq N
$$

Policy 4 (Isolation energy and remainder) does not always satisfy coalitional rationality since cost of the coalitions is not considered in Equation 6 (Theorem D.1). Policy 5 (Shapley value) does not always satisfy coalitional rationality. However, if the resulting game based on the energy consumption values $E(S)$ is concave, Policy 5 satisfies the property [Rosenbusch 2011]. 
Finally, Policy 7 (Least square nucleolus) is not always coalitionally rational, but other works have shown that it is always contained in an approximation of the core (least-core), which describes approximately stable outcomes [Maschler et al. 1992; Molina and Tejada 2000]. Additionally, Policy 7 is the only solution ensuring that on average all entities are equally treated if each entity evaluates the excess of the coalitions that the entity belongs to: $\sum_{S \mid i \in S} e(S, \pi)=\sum_{S \mid j \in S} e(S, \pi), \forall i, j \in N$. The egalitarian principle towards coalitions becomes egalitarian towards the entities too, and thus this solution contributes to stability.

5.2.5 Entity desirability. Policies 1, 2, 3, 4 and 6 always satisfy entity desirability. Our proofs for entity desirability can be found in section $\mathrm{E}$ of the appendix.

Policy 1 (Equal division) trivially satisfies it since the policy always prescribes $\pi_{i}=\pi_{j}$ (Theorem E.1). Policies 2 and 4 (Proportional to isolation and Isolation energy and remainder) always satisfy entity desirability. A more desirable entity has a lower (or at least equal) isolated consumption $E(\{i\})$ than a less desirable entity, and thus Equations 4 and 6 prescribe always a lower (or at least equal) apportionment (Theorems E.2 and E.4 respectively). Policy 3 (Marginal contribution) always satisfy entity desirability since a more desirable entity has a lower marginal contribution (Theorem E.3).

Policy 5 (Shapley value) does not always satisfy the property for the general case, but the policy will always satisfy entity desirability when the game is subadditive [Rosenbusch 2011].

Policy 7 (Least square nucleolus) does not always satisfy entity desirability, but instead it satisfies an attractive property: aggregated entity desirability ${ }^{1}$. Instead of comparing the marginal contribution of two entities to each coalition, this property compares the total sum of the marginal contributions to all coalitions and prescribes a smaller cost if the sum of an entity's contribution is smaller than or equal to another entity's contribution [Ruiz et al. 1996]:

$$
\begin{gathered}
\text { if } \sum_{S \mid i, j \notin S} E(S \cup\{i\})-E(S) \leq \sum_{S \mid i, j \notin S} E(S \cup\{j\})-E(S) \\
\text { then } \pi_{i} \leq \pi_{j} \text { for all } i, j \in N
\end{gathered}
$$

The approach of Policy 7 considers all the coalitions equally important.

Finally, the surrogate-based policies $(8,9$ and 10) do not always satisfy entity desirability since less activity does not necessarily mean lower energy consumption (Theorems E.5, E.6 and E.7).

\subsection{Summary of the fairness insights}

Table II shows that Policy 6 (Nucleolus) provides the strongest fairness since it satisfies all properties. The intuition is that Policy 6 is defined to minimise the unhappiness of the most unhappy entity in the system.

Policy 5 (Shapley value) is an excellent and fair alternative provided that the system energy function $E$ is always subadditive for the system. If subadditivity does not hold, the only advantage of this policy compared to the other ones is that it satisfies the dummy player property. Thus, subadditivity is a precondition for a system to employ the full potential of the Shapley value policy.

Policy 1 (Equal division) and Policy 3 (Marginal contribution) satisfy only symmetry and entity desirability, which makes other policies such as Policy 2 (Proportional to isolation) more attractive. Policy 2, is preferred to Policy 4 (Isolation energy and remainder) since Policy 2 additionally satisfies the subset case of dummy player when the contribution of an entity is 0 . Policy 2 is an attractive choice given the symmetry, individual rationality and entity desirability criteria, which provide incentives and a notion of fairness based on comparing two entities in the system.

Policy 7 (Least Square Nucleolus) is very interesting alternative given its more egalitarian approach to fairness and the additional fairness properties that it satisfies compared to Policy 5 or 6 . In addition, all

\footnotetext{
${ }^{1}$ This property is called Average Marginal Contribution Monotonicity, but we rename it to keep term consistency in the evaluation.

Authors' preprint
} 
coalitions are equally treated by the policy (i.e., all the complaints have the same weight) and it contributes to stability.

The detailed analysis of the above 10 policies pinpoints that surrogate policies do not provide guarantees in terms of fairness from the energy perspective defined as the 5 criteria. If the antecedents of the fairness properties are defined in terms of the activity function $a$ instead of $E$, e.g., for symmetry $a(S \cup\{i\})=$ $a(S \cup\{j\})$ for all $S \subseteq N \backslash\{i, j\}$, Policies 8 (Proportional to activity) and 9 (Time slicing) satisfy activity-based symmetry and entity desirability. Nevertheless, the two criteria providing incentives (individual rationality and coalitional rationality) inherently require energy related concepts, and thus activity needs to be correlated to energy, otherwise surrogate-based policies can provide misleading incentives.

\section{SUMMARY OF THE ANALYSIS AND DISCUSSION}

In this section we provide an overview of the presented analysis considering the different evaluation criteria and the identified trade-offs. Section 6.1 summarises the major trade-offs between fairness and the other evaluation criteria. Section 6.2 discusses the importance of analysing the system energy function $E$, and finally section 6.3 provides some high-level guidelines to select an energy apportionment policy given the purpose of the apportionment.

\subsection{General trade-offs}

The major trade-offs identified in the analysis are the following:

Fairness vs. information type: We have distinguished between energy-based and surrogate-based energy apportionment policies based on the type of input information. Surrogate information (e.g., resource access time or data sent) is in general easily accessible compared to energy-based information which usually needs to be estimated.

However, we have shown that some fairness properties (dummy player, individual rationality and coalitional rationality) cannot be satisfied by a surrogate-based policy. In addition, if the surrogate information is not highly correlated with the energy information, the surrogate policies will not always satisfy the other fairness properties (symmetry and entity desirability). Table II reflects this issue showing that none of the surrogate-based policies satisfies any of the 5 fairness properties in the general case.

Fairness vs. computational complexity: The amount of input information is tightly related to the computational complexity of a policy, which leads to a trade-off between fairness and computational complexity.

Policy 1 (Equal division) provides the lowest complexity but also weak fairness. Policies 2, 3 and 4 present low information requirement (a single value per entity) leading to a low computational complexity, but none of these policies satisfies the 5 fairness properties. We identified Policy 2 (Proportional to isolation) as an interesting policy in the trade-off between fairness and computational complexity: it provides reasonable fairness (providing symmetry and entity desirability) as well as incentives (individual rationality) in linear time.

Surrogate-based policies (Policies 8, 9 and 10) are not very attractive since they do not satisfy any fairness property and the computation requirement is still similar to Policies 2,3 and 4 .

Satisfying the additional fairness properties requires more input information (e.g., to compare coalitions) leading to higher computational complexity. Policy 6 (Nucleolus) satisfies all fairness properties, and Policies 5 and 7 (Shapley value and Least square nucleolus) have a well defined notion of fairness. However, the size of the input grows exponentially with the number of entities for the game-theoretic policies. Moreover, coalitional rationality (satisfied by Policy 6) relates to an inherently hard problem. Determining whether there exists a set of coalitionally rational apportionments is NP-complete [Conitzer and Sandholm $2003 ; 2006]$. 
Thus, the computational cost of satisfying all fairness properties is high, and lowering the computational cost requires selecting a smaller set of fairness properties. Alternatively, one could apply a high complexity approach to a problem with a small set of inputs. Moreover, as pointed out in section 4.2.2, there are efficient algorithms to compute the game-theoretic policies for restricted classes of games.

To sum up, fairness comes at at cost of having to measure or estimate energy consumption as well as computational complexity.

\subsection{Properties of the system energy function}

The system energy function $E$ models the energy consumption of the system and its values are either measured or estimated. In section 2.3 we introduced some common properties used to classify $E$ (e.g., subadditive or concave). Knowing the class of $E$ is highly valuable for Policy 5 (Shapley value) since it satisfies relevant fairness properties only for some of the $E$ categories.

While subadditivity is often an assumption for some problems in the game theory literature [Shapley 1953; Engevall et al. 1998], it is difficult to say how likely it is that the system function $E$ is subadditive for energy systems. If $E$ is subadditive, the energy consumption of two coalitions (resp. entities) together is not higher than the sum of their isolated consumption. This implies that being together in the system is always advantageous, which implies that the interaction of the entities in the system is efficient. While analysing whether an instance of $E$ is subadditive is intuitively system dependent, we provide some illustrative examples that result in a subadditive $E$ and a non-subadditive $E$ respectively:

Subadditive: Consider a system similar to the one presented in the introduction section, where major contribution to the energy consumption is due to an inactivity timer triggered after performing some activity. For example, a Secure Digital card (SD card) consumes energy for some seconds after each write/read operation [Pathak et al. 2012]. If two applications use the SD card concurrently, the total energy is smaller than sum of the stand-alone energy of the applications (overlapping energy tails). If the applications use it separately, the consumption is simply the sum of the stand-alone energy. Thus, the system is subadditive. Other examples following a similar intuition are a water kettle or a TV [Reinhardt et al. 2012b], where the usage overlap by several users intuitively results in a subadditive $E$.

Non-subadditive: Consider the $3 \mathrm{G}$ wireless interface which is often characterised by two main consuming states depending on the utilisation [Vergara et al. 2014b]. The drivers of some WiFi interfaces use a similar mechanism [Pyles et al. 2012]. Small amounts of data are sent in a low consuming state, but when the data sent for a period of time is higher than a given threshold, the interface is moved to a high energy state. The consumption of two processes sending small amounts of data together can lead to the high energy state, whereas sending data separately would result in a lower consumption due to using the low energy state separately. Thus, this system is not always subadditive.

Consider another simple example from the energy-efficient building area, a refrigerator, whose compressor is activated periodically in order to maintain the desired temperature in the hysteresis region [Cheng et al. 2012]. Opening the refrigerator increases the need of starting the compressor earlier. For this system, the result of two people opening the refrigerator separately can easily result in a non-subadditive $E$ since the entities alone would consume less than together.

Concavity implies subadditivity, and hence, from the discussion above, it follows that some real-world examples of $E$ may not satisfy concavity.

To sum up, we believe that further work is needed to analyse the properties of $E$ for different types of systems. It is also interesting to analyse the properties of $E$ for complex systems composed of different subsystems (e.g., a mobile device has a SD card as well as some wireless interfaces). 


\subsection{Selection guidelines}

In this section we outline the most significant criteria that impact the selection of a policy for energy apportionment. Based on our analysis and the previous discussion, the following criteria serve as guidelines in order to select a policy:

Surrogate vs. energy-based policy: Where applicable, we believe that energy-based policies are more appropriate than surrogate-based ones. Highly accurate energy models and measurement techniques are already available for many systems and obtaining better estimates of the $E$ values is an ongoing work. This will facilitate the information acquisition for the apportionment.

Number of entities and real-time requirements: The number of entities in the system plays an important role on the computational complexity, and this can drastically impact the timing requirements for energy apportionment. In general, complex algorithms are not fit for large number of entities, especially in real-time applications of apportionment. However, they may make sense in offline analysis settings. Analysing the running time of the apportionment policy implementations in different systems as well as their energy cost is an interesting future direction.

The purpose of apportionment: We classify the purpose of apportionment into three main categories: informing, profiling and incentivising.

Informing: When the purpose of the energy apportionment is simply to inform about the overall energy consumption of the entities (e.g., rank the most consuming entities), there is no strict need for a complex policy. We believe that Policy 2 (Proportional to isolation) is a good fit for this purpose since it includes a fairness notion where each entity is compared in a one-to-one fashion (symmetry, null player and entity desirability).

Profiling: This applies when the purpose of the apportionment is to accurately profile the energy usage (via testing) by entities in a system, e.g., for improving the efficiency of applications or software optimisation. Symmetry and entity desirability are necessary properties for this purpose and the dummy player property is desirable. Table II shows that Policy 5 (Shapley value) and Policy 6 (Nucleolus) are good choices for this purpose.

Incentivising: Energy apportionment can provide incentives to reduce the energy consumption, especially when the entities of the system can influence their energy consumption. Basic incentives can be realised by selecting a policy that satisfies individual rationality, such as the simple Policy 2 (Proportional to isolation). It provides incentives to reduce the stand-alone consumption of an entity, since this will be apportioned less than its stand-alone consumption when the system is efficient. For example, it can incentivise application developers to reduce the stand-alone energy of their applications.

Satisfying coalitional rationality is recommended when the purpose is to incentivise entities to efficiently cooperate in the system in addition to reduce their stand-alone consumption. For example, two applications can be incentivised to reduce their consumption together (e.g., by synchronising transmission patterns) because they are apportioned no more than their stand-alone consumption, but also no more than their consumption together. Coalitional rationality is desirable in systems where multiple dynamic entities can join or leave the system (e.g., device-to-device clusters in 5G [Bianzino et al. 2014; Boccardi et al. 2014]), where incentives are the key to cooperation.

Thus, a system can benefit from a strong incentive structure of Policy 6 (Nucleolus) which satisfies individual and coalitional rationality, at the cost of computational complexity. A more egalitarian fairness notion towards all coalitions of Policy 7 (Least square nucleolus) is also an interesting alternative when users are involved (e.g., energy-efficient buildings).

Finally, if the subadditivity and concavity of $E$ can be shown, Policy 5 (Shapley value) can also provide incentives. 
Thus, depending on the purpose of energy apportionment and the system specifics some policies can be a better fit than others. We have identified that Policy 2 (Proportional to isolation) provides an interesting tradeoff between computational complexity and fairness while still providing basic incentives. Policy 6 (Nucleolus) and Policy 7 (Least square nucleolus) are the most interesting properties in order to provide stronger incentives, and Policy 5 (Shapley value) and Policy 6 (Nucleolus) are a good fit to profile systems. Finally, it is clear that analysing $E$ is crucial for employing Policy 5 (Shapley value).

\section{RELATED WORKS}

Our work has studied the energy apportionment problem from a perspective not adopted before. In section 7.1 we describe how different works have practically approached energy apportionment and describe their context as well as the implemented policy. In addition, section 7.2 provides a brief overview of the area of energy management and how energy apportionment plays a highly relevant role on it.

\subsection{Energy accounting}

This section is devoted to describe the application and implementation of the presented energy apportionment policies. We briefly explain each approach, analyse and report which policies they employ. The works are categorised in four different contexts: processing and operating systems, wireless sensor networks, energyefficient buildings and mobile devices.

7.1.1 Processing and operating systems. PowerScope [Flinn and Satyanarayanan 1999b] maps energy consumption to program structure by employing physical power measurements combined with kernel level system activity information. The work studies a single-core CPU, and thus attributes a process the energy consumed when the process is being executed. Since a single process can be executed simultaneously, the process is allocated its consumption based on the time slice when it was scheduled (similar to Policy 9 - Time slicing). This work does not consider cases where the activity of entities in the system is not instantaneously reflected in the power trace (e.g., energy tails).

A follow up work by Bellosa [Bellosa 2000] proposes Joule Watcher, a similar event-driven resource profiler to attribute energy consumption of CPU and memory to individual threads. Even though the work does not adress the problem of multi-entity apportionment, it refers to surrogate information such as performancemonitoring information.

Neugebauer and McAuley [2001] argue that energy accounting of shared resources is a requirement for energy management. They discuss the difficulties in estimating each entity's consumption due to asynchronous energy overheads (e.g., energy tails). They suggest that the apportionment should be done in proportion to utilisation-based surrogates, i.e., Policy 8 (Proportional to activity). Example of surrogates are the access time to a resource, number of pixels used in a display or amount of data exchanged with the network.

ECOSystem [Zeng et al. 2002b] is an energy-centric operating system which employs energy accounting for the CPU, disk and network card. The running time of the process is used to apportion the CPU consumption assuming that the CPU consumes a fixed amount of power (Policy 9). The active access of the disk is computed using surrogates when write or read operations occur. The consumption due to asynchronous operation (e.g., spin-up, spinning and spin-down) is proportionally divided by the tasks that employed it between the spin-up and spin-down events. This is similar to Policy 9 (Time slicing) considering the spin-up and spin-down as events. Finally, the energy consumption of the network interface is attributed based the amount of data transmitted and received.

Ryffel et al. [2009] and $\mathrm{LEA}^{2} \mathrm{P}$ [Ryffel 2009] extend the idea of PowerScope to multi-core system and propose Policy 3 (Marginal contribution) as fair without any formal justification. The system employs power measurements of different hardware elements (e.g., CPU, GPU or hard drive) combined with indirect information based on performance counters. However, they mention that obtaining the energy consumption values 
for Policy 3 is not feasible for some system components such as CPU since processes run simultaneously. Estimating the energy consumption of a process in isolation (in a single CPU) is proposed to overcome the limitation, and thus Policy 2 (Proportional to isolation) is employed to estimate the apportionment of each process for the multi-core processor.

Joulemeter [Kansal et al. 2010] is a system to attribute the energy consumption of a cloud server to the running virtual machines (VMs). The energy consumption of each VM is estimated based on measurementbased energy models. It is argued that the apportionment is correct if the sum of the estimated energy is equal to the total measured energy (i.e., efficiency), which is not always the case. They consider the energy in isolation as the apportionment for each VM (similar to Policy 2).

7.1.2 Wireless sensor networks. Quanto [Fonseca et al. 2008] is an energy profiler for network nodes which combines energy metering hardware and program activity information to quantify the energy consumption for user defined activities. They use the notion of activity as a logical set of operations whose resource usage is grouped together. An entity can adopt the same notion in our work.

Quanto estimates the energy breakdown of the different components and then system quantifies the resource consumption of the activities. A single-activity device is considered leading to a straightforward apportionment, but the authors mention that the apportionment for a multiple-activity device is a policy decision. They preliminary select to divide the energy consumption equally, i.e., in a similar way to Policy 1.

Kellner [2010] considers the context of dynamic sensor networks where various applications can query a sensor network at any point in time. The work proposes an online energy accounting system employing measurement-based finite state machine (FSM) models to attribute energy consumption to tasks. Since energy consumption does not only depend on the active usage (e.g., energy tails or between uses), the author identifies several ways to divide the remainder energy. Policy 4 (Isolation energy and remainder) is applied to message reception where the energy consumption of the messages that cannot be attributed directly to any task is equally shared. Policy 9 (Time slicing) is used to divide the energy consumption of the micro controller considering active or inactive tasks.

7.1.3 Energy-efficient buildings. A first and influential work by Hay and Rice [Hay and Rice 2009] formulate the problem of attributing the energy consumption of a building or organisation to individual users. They argue that it can provide incentives to reduce the total consumption and identify two requirements that every policy should satisfy: completeness and accountability. The first one refers to efficiency, whereas the second states that actions of a user should have a maximal effect on their own share and a minimal on the rest of users. The entity desirability property is related to accountability, where a more consuming entity will get prescribed a higher energy consumption.

Their work proposes Policy 1 (Equal division) as the most basic policy. This is further extended using occupancy time as a surrogate of energy resulting in a version of Policy 8 (Proportional to activity). Policy 4 (Isolation energy and remainder) is then proposed by first calculating the personal load of a user and dividing the rest evenly. Their efforts focus on accurately estimating the personal load employing sensors.

Similarly, WattShare [Thakur et al. 2014] seeks to attribute the energy consumption of a shared building to individual occupants (personal apportionment) using Policy 9 (Time slicing). Considering only the total energy consumption measured at the smart meter, their work proposes to employ information from smartphone sensors (e.g., WiFi signal strength or microphone data) carried by the occupants to perform the apportionment.

Tsao et al. [2014] investigate how to attribute the energy consumption of centralised heating, ventilation and air conditioning system to different rooms in a building. They propose to employ Policy 2 (Proportional to isolation) and argue that it satisfies efficiency and accountability. There is no evidence showing that their apportionment approach satisfies the two properties. 
7.1.4 Mobile devices. Pushed by short battery lifetimes energy profiling has attracted a great attention in mobile devices to improve software energy efficiency. Our previous work EnergyBox [Vergara et al. 2014b] develops a FSM-based energy model for wireless interfaces. By isolating the traffic of an application the energy efficiency of the applications is studied in isolation using EnergyBox [Vergara et al. 2014a; Almquist et al. 2015]. Other studies perform a similar simplifying assumption to profile the energy consumption due to communication [Qian et al. 2011; Rice and Hay 2010] which is similar to Policy 2 (Proportional to isolation).

Similarly, WattsOn [Mittal et al. 2012] is a system to estimate the energy consumption by an application at the development environment which reuses and extends previously developed FSM-based energy models. This work considers that the energy consumption of the application is the total energy consumption of the system when only the application is running (i.e., similar to Policy 2). Zhang et al. [2010] develop activity-based power models (FSM and linear functions) using measurements in order to attribute the energy consumption of each component to the different processes in the system. Their work does not consider the asynchronous energy consumption such as energy tails.

Bugu [Li et al. 2014] is an application level service which aims to profile the applications running in client devices by sending consumption statistics to a server. The considered apportionment policy is that an application's consumption is the energy consumption in isolation $(E(\{i\}))$ based on their power model (similar to Policy 2), which does not always satisfy efficiency.

The characterisation of graphical user interfaces and display energy consumption from hardware, software and application perspective is performed with a focus on optimising the energy consumption of GUI applications and GUI platforms [Zhong and Jha 2003]. The work uses Policy 3 (Marginal contribution) to obtain the energy contribution of GUIs.

Eprof [Pathak et al. 2012] is an energy profiler for smartphones to support efficient application development. It employs system call-based resource usage information and FSM-based models to attribute the energy consumption to applications (and activities within the application). They discuss different alternatives to attribute the energy consumption of energy tails such as equally (Policy 1) or proportionally dividing the energy consumption among the consuming entities (Policy 2). Finally, they propose the last-trigger policy (Policy 10 - Last active in our work) in order to incentivise the developer to batch energy events. In our work we formalised their policy proposal to analyse its properties.

The recent work by Dong et al. [2014] is the first work considering the application of cooperative game theory in the context of mobile energy accounting. They argue that the Shapley value (Policy 5) is the ground truth for the apportionment problem in mobile systems and provide an approximation of it by approximating the game. Using physical power measurements they define every 10-millisecond interval as a game where the energy consumption needs to be attributed. By considering these short intervals they attempt to observe the consumption of different coalitions (i.e., observing intervals where only some entities are active) and employ a combination of the observed coalitions with hardware states (for WiFi and CPU) to reduce the variance of the observations. The unobserved coalitions are statistically estimated from historical data. Their results show that other accounting methods differ significantly from their approach.

Recent work has also focused on identifying energy hungry applications by collaboratively collecting usage information from many devices and employing statistical techniques [Oliner et al. 2013; Chandra et al. 2013]. These works provide a limited level of energy accounting due to the coarse granularity of the approach that reduces the possibilities of using them in energy management.

Compared to the previous works we focus on analysing the fundamental aspects of energy apportionment policies rather than application aspects of them. Our work provides an overview of the energy apportionment problem in different research areas in order to unify efforts towards energy-efficiency.

We show that fairness is a relevant criterion and pinpoint that, as in cooperative game theory, there is no best solution in general. Additionally, we propose the usage of two apportionment policies with strong 
fairness notions which have not previously been used in the described areas. Finally, we provide guidelines to select a policy based on the information requirement, computational complexity and fairness properties and suggest that the analysing structure of the $E$ function for a system is decisive.

\subsection{Energy management}

Efficiently managing system resources in terms of energy requires to control and determine the energy expenditure of the system and the entities. Energy accounting, and thus energy apportionment too, are key elements to determine the energy consumption of the entities. This section describes approaches considering energy allocation to competing entities in the system, mostly from the operating system and mobile device literature. These rely on energy accounting and apportionment policies.

The first energy-aware operating system concepts and attempts date from late 90s [Ellis 1999; Neugebauer and McAuley 2001]. Ellis [1999] advocates that energy consumption should be considered as a first class resource while still meeting performance requirements. Since then, the main approach in energy management is to devise different strategies to control the energy consumption of the system by limiting the energy usage of entities in the system (e.g., applications).

Budget-based energy management (BBEM) allocates an energy budget to entities in the system (e.g., applications) and these will not run if they run out of budget. Setting a target lifetime is also a recurring trend. Nemesis [Neugebauer and McAuley 2001], ECOSystem [Ellis 1999; Zeng et al. 2002b; 2003] and Odyssey [Flinn and Satyanarayanan 1999a; 2004] are examples of early operating systems that propose adaptive applications which can tradeoff QoS for lower consumption. These works also pinpointed the need for energy accounting. For example, Odyssey uses PowerScope [Flinn and Satyanarayanan 1999b] to build a system's power model which and employs Policy 8 as apportionment policy. More recently BBEM has been adopted and extended by several works to extend the battery lifetime of mobile devices (e.g., Cinder [Rumble et al. 2009; Roy et al. 2011]) The complexity of BBEM lies on devising a strategy to define the budgets and the replenishment rate to overcome the potential QoS and QoE burden on the user when applications run out of budget. Prioritising some applications [Cho et al. 2014], limiting background applications [Chandra et al. 2013] or exploiting context information (e.g., user location) [Chu et al. 2011; Vallina-Rodriguez and Crowcroft 2011] are some examples of recent proposals for energy management.

Finally, the theoretical work by Dong [2013] formulates energy management as a utility optimisation problem: schedule the different processes to maximise the aggregate utility of all system processes under the energy capacity constraint. BBEM is compared to a novel energy management strategy which dominates by optimal utility value.

To sum up, energy is a vital resource and efficiently managing system resources from an energy perspective is an ongoing activity. Selecting an appropriate energy apportionment policy for a system is important for efficient energy accounting and energy management.

\section{CONCLUSIONS AND FUTURE WORK}

In this paper we categorised, compared and analysed a total of 10 different energy apportionment policies in terms of required information, computational complexity and fairness. Among the 10 policies, we formalised 3 policies not described mathematically earlier, and proposed two novel policies based on cooperative game theory.

Our work shows that cooperative game theory provides an excellent framework and solutions for addressing the energy apportionment problem, and that selecting an appropriate policy plays a role in providing incentives for energy efficiency to the entities in the system.

Unfortunately, there is no known ground truth for the general apportionment problem and therefore energy-based policies are no better than surrogate-based ones. However, we have pinpointed that providing incentives and stability requires energy related concepts and that an energy apportionment policy needs to 
reflect the inner workings of the system leading to energy consumption. Thus, employing the system energy function in the apportionment seems a more reasonable alternative.

Our results demonstrate that there is a trade-off between fairness and the other evaluation criteria. The strongly fair policies based on game theory have the highest information requirement and computational complexity, which can be less practical in some contexts (e.g., high number of entities in the system or timing requirements). Depending on the context, a simpler policy like Policy 2 (Proportional to isolation) can achieve a satisfactory compromise by sacrificing some fairness properties but drastically reducing complexity and still providing basic incentives. Further work can be done to identify how to satisfy a set of fairness properties with minimum complexity

Finally, the characteristics and properties of the system energy function should be analysed since some policies assume a certain properties (e.g., Shapley value) and sometimes different policies can lead to identical results. In the latter case selecting the simplest policy among the equivalent ones is recommended.

The future work directions include applying the above insights in systems from different contexts and empirically studying the impact of the incentives on energy efficiency. Developing means to measure or better approximate the system function for different is a key capability which can provides insights about the structure.

\section{Acknowledgment}

This work was supported by the Swedish national graduate school in computer science (CUGS). The authors wish to thank Holger I. Meinhardt and Artus Ph. Rosenbusch for their insightful comments about game theory.

\section{REFERENCES}

Almquist, M., Almquist, V., Vergara, E. J., And Nadjm-Tehrani, S. 2015. Communication energy overhead of mobiles games. In Proceedings of the 2nd Workshop on Mobile Gaming. MobiGames '15. ACM, 1-6.

Athukorala, K., Lagerspetz, E., von Kügelgen, M., Jylhä, A., Oliner, A. J., Tarkoma, S., and Jacucci, G. 2014. How Carat Affects User Behavior: Implications for Mobile Battery Awareness Applications. In Proceedings of the ACM Conference on Human Factors in Computing Systems. CHI '14. ACM, 1029-1038.

Balasubramanian, N., Balasubramanian, A., and Venkataramani, A. 2009. Energy consumption in mobile phones: A measurement study and implications for network applications. In Proceedings of the 9th ACM SIGCOMM Conference on Internet Measurement Conference. IMC '09. ACM, 280-293.

Bellosa, F. 2000. The benefits of event-driven energy accounting in power-sensitive systems. In Proceedings of the 9th Workshop on ACM SIGOPS European Workshop: Beyond the PC: New Challenges for the Operating System. EW 9. ACM, $37-42$.

Bertran, R., Becerra, Y., Carrera, D., Beltran, V., Gonzalez Tallada, M., Martorell, X., Torres, J., And Ayguade, E. 2010. Accurate energy accounting for shared virtualized environments using PMC-based power modeling techniques. In IEEE/ACM International Conference on Grid Computing. 1-8.

Bianzino, A. P., Asplund, M., Vergara, E. J., And Nadjm-Tehrani, S. 2014. Cooperative proxies: Optimally trading energy and quality of service in mobile devices. Computer Networks 75, Part A, $297-312$.

Boccardi, F., Heath, R., Lozano, A., Marzetta, T., and Popovski, P. 2014. Five disruptive technology directions for 5g. Communications Magazine, IEEE 52, 2, 74-80.

Castro, J., Gómez, D., And Tejada, J. 2009. Polynomial Calculation of the Shapley Value Based on Sampling. Comput. Oper. Res. 36, 5, 1726-1730.

Chandra, R., Fatemieh, O., Moinzadeh, P., Thekkath, C. A., and Xie, Y. 2013. End-to-end energy management of mobile devices. Tech. Rep. MSR-TR-2013-69, Microsoft Research. July.

Cheng, Y., Chen, K., Zhang, B., Liang, C.-J. M., Jiang, X., And ZhaO, F. 2012. Accurate real-time occupant energyfootprinting in commercial buildings. In Proceedings of the Fourth ACM Workshop on Embedded Sensing Systems for Energy-Efficiency in Buildings. BuildSys '12. ACM, 115-122.

Cho, J., Woo, Y., Kim, S., And Seo, E. 2014. A battery lifetime guarantee scheme for selective applications in smart mobile devices. Consumer Electronics, IEEE Transactions on 60, 1, 155-163.

Authors' preprint 
Chu, D., Kansal, A., Liu, J., And Zhao, F. 2011. Mobile apps: It's time to move up to condos. In Proceedings of the 13th USENIX Conference on Hot Topics in Operating Systems. HotOS'13. USENIX Association, Berkeley, CA, USA, 16-16.

Conitzer, V. And Sandholm, T. 2003. Complexity of determining nonemptiness of the core. In Proceedings of the 4th ACM Conference on Electronic Commerce. EC '03. ACM, New York, NY, USA, 230-231.

Conitzer, V. And SAndholm, T. 2006. Complexity of constructing solutions in the core based on synergies among coalitions. Artificial Intelligence 170, 67, $607-619$.

Deng, X. and Papadimitriou, C. H. 1994. On the complexity of cooperative solution concepts. Math. Oper. Res. 19, 2, $257-266$.

Dong, M. 2013. Energy Accounting and Optimization for Mobile Systems. Ph.D. thesis, Rice University.

Dong, M., LAN, T., And Zhong, L. 2014. Rethink energy accounting with cooperative game theory. In Proceedings of the 20th Annual International Conference on Mobile Computing and Networking. MobiCom '14. ACM, 531-542.

Drechsel, J. 2010. Selected topics in cooperative game theory. In Cooperative Lot Sizing Games in Supply Chains. Lecture Notes in Economics and Mathematical Systems Series, vol. 644. Springer, 5-39.

Drechsel, J. AND Kimms, A. 2010. Computing core allocations in cooperative games with an application to cooperative procurement. International Journal of Production Economics 128, 1, 310 - 321.

ElLiS, C. 1999. The case for higher-level power management. In Proceedings of the Seventh Workshop on Hot Topics in Operating Systems, 1999. IEEE, 162-167.

Engevall, S., Göthe-Lundgren, M., And VÄrbrand, P. 1998. The traveling salesman game: An application ofcost allocation in a gas and oil company. Annals of Operations Research 82, 0, 203-218.

Faigle, U., Kern, W., And Kuipers, J. 1998. Computing the Nucleolus of Min-cost Spanning Tree Games is NP-hard. Int. J. Game Theory 27, 3, 443-450.

Fatima, S. S., Wooldridge, M., And Jennings, N. R. 2008. A Linear Approximation Method for the Shapley Value. Artif. Intell. 172, 14, 1673-1699.

Fiestras-Janeiro, M., García-Jurado, I., And Mosquera, M. A. 2011. Cooperative games and cost allocation problems. TOP 19, 1, 1-22.

Flinn, J. And Satyanarayanan, M. 1999a. Energy-aware adaptation for mobile applications. SIGOPS Oper. Syst. Rev. 33, 5, $48-63$.

Flinn, J. And Satyanarayanan, M. 1999b. PowerScope: A Tool for Profiling the Energy Usage of Mobile Applications. In Proceedings of the 2nd IEEE Workshop on Mobile Computer Systems and Applications. WMCSA '99. IEEE.

Flinn, J. and Satyanarayanan, M. 2004. Managing battery lifetime with energy-aware adaptation. ACM Trans. Comput. Syst. 22, 2, 137-179.

Fonseca, R., Dutta, P., Levis, P., And Stoica, I. 2008. Quanto: Tracking energy in networked embedded systems. In Proceedings of the 8th USENIX Conference on Operating Systems Design and Implementation. OSDI'08. USENIX Association, Berkeley, CA, USA, 323-338.

Hay, S. And Rice, A. 2009. The case for apportionment. In Proceedings of the First ACM Workshop on Embedded Sensing Systems for Energy-Efficiency in Buildings. BuildSys '09. ACM, 13-18.

Kansal, A., Zhao, F., Liu, J., Kothari, N., And Bhattacharya, A. A. 2010. Virtual machine power metering and provisioning. In Proceedings of the 1st ACM Symposium on Cloud Computing. SoCC '10. ACM, New York, NY, USA, 39-50.

Kellner, S. 2010. Flexible Online Energy Accounting in TinyOS. In Proceedings of the 4th International Workshop on Real-World Wireless Sensor Networks. LNCS Series, vol. 6511. Springer, 62-73.

Lee, E. A. 1999. Modeling Concurrent Real-time Processes Using Discrete Events. Ann. Softw. Eng. 7, 1-4, 25-45.

Lemaire, J. 1984. An application of game theory: Cost allocation. ASTIN Bulletin 14, 61-81.

Li, Y., Chen, H., And Shi, W. 2014. Power behavior analysis of mobile applications using bugu. Sustainable Computing: Informatics and Systems 4, 3, $183-195$.

Maschler, M., Peleg, B., And Shapley, L. S. 1979. Geometric Properties of the Kernel, Nucleolus, and Related Solution Concepts. Math. Oper. Res. 4, 4, 303-338.

Maschler, M., Potters, J., And Tijs, S. 1992. The general nucleolus and the reduced game property. Int. J. Game Theory 21, 1, 85-106.

Masuya, S. AND InUiguchi, M. 2009. Toward the theory of cooperative games under incomplete information. In Proceedings of the 6th International Conference on Modeling Decisions for Artificial Intelligence. MDAI '09. Springer-Verlag, 102-113.

McCAIn, R. A. 2013. Value Solutions in Cooperative Games. World Scientific Publishing Company.

Megiddo, N. 1978. Computational complexity of the game theory approach to cost allocation for a tree. Math. Oper. Res. 3, 3, $189-196$. 
Misra, V., Ioannidis, S., Chaintreau, A., And Massoulié, L. 2010. Incentivizing Peer-assisted Services: A Fluid Shapley Value Approach. In Proceedings of the ACM SIGMETRICS International Conference on Measurement and Modeling of Computer Systems. SIGMETRICS '10. ACM, 215-226.

Mittal, R., Kansal, A., And Chandra, R. 2012. Empowering Developers to Estimate App Energy Consumption. In Proceedings of the 18th Annual International Conference on Mobile Computing and Networking. Mobicom '12. ACM, 317-328.

Molina, E. And TejadA, J. 2000. The least square nucleolus is a general nucleolus. Int. J. Game Theory 29, 1, 139-142.

Moretti, S. And Patrone, F. 2008. Transversality of the Shapley value. TOP 16, 1, 1-41.

Neugebauer, R. And McAuley, D. 2001. Energy Is Just Another Resource: Energy Accounting and Energy Pricing in the Nemesis OS. In Proceedings of the Eighth Workshop on Hot Topics in Operating Systems. HOTOS '01. IEEE.

Oliner, A. J., Iyer, A. P., Stoica, I., Lagerspetz, E., and Tarkoma, S. 2013. Carat: Collaborative energy diagnosis for mobile devices. In Proceedings of the 11th ACM Conference on Embedded Networked Sensor Systems. SenSys '13. ACM, New York, NY, USA, 10:1-10:14.

Papathanasiou, A. And Scott, M. 2003. Energy efficiency through burstiness. In Fifth IEEE Workshop on Mobile Computing Systems and Applications. 44-53.

Pathak, A., Hu, Y. C., And Zhang, M. 2012. Where is the Energy Spent Inside My App?: Fine Grained Energy Accounting on Smartphones with Eprof. In Proceedings of the 7th ACM European Conference on Computer Systems. EuroSys '12. ACM, $29-42$.

Petersen, J. E., Shunturov, V., Janda, K., Platt, G., and Weinberger, K. 2007. Dormitory residents reduce electricity consumption when exposed to real-time visual feedback and incentives. International Journal of Sustainability in Higher Education 8, 16-33.

Polkowski, L. And Araszkiewicz, B. Elsevier. 2003. A rough set approach to estimating the game value and the shapley value from data. Electron. Notes Theor. Comput. Sci. 82, 4, $219-227$.

Puerto, J. And Perea, F. 2013. Finding the nucleolus of any n-person cooperative game by a single linear program. Computers and Operations Research 40, 10, 2308-2313.

Pyles, A. J., Qi, X., Zhou, G., Keally, M., and Liu, X. 2012. Sapsm: Smart adaptive 802.11 psm for smartphones. In Proceedings of the 2012 ACM Conference on Ubiquitous Computing. UbiComp '12. ACM, New York, NY, USA, 11-20.

Qian, F., Wang, Z., Gerber, A., Mao, Z., Sen, S., And Spatscheck, O. 2011. Profiling resource usage for mobile applications: A cross-layer approach. In Proceedings of the 9th International Conference on Mobile Systems, Applications, and Services. MobiSys '11. ACM, 321-334.

Reinhardt, A., Baumann, P., Burgstahler, D., Hollick, M., Chonov, H., Werner, M., and Steinmetz, R. 2012a. On the accuracy of appliance identification based on distributed load metering data. In Sustainable Internet and ICT for Sustainability (SustainIT), 2012. IEEE, 1-9.

Reinhardt, A., Baumann, P., Burgstahler, D., Hollick, M., Chonov, H., Werner, M., and Steinmetz, R. 2012b. On the accuracy of appliance identification based on distributed load metering data. In Sustainable Internet and ICT for Sustainability (SustainIT), 2012. 1-9.

Rice, A. And Hay, S. Elsevier. Dec. 2010. Measuring mobile phone energy consumption for 802.11 wireless networking. Pervasive Mobile Computing 6, 6, 593-606.

Rosenbusch, A. P. 2011. Fairness Considerations in Cooperative Games. Ph.D. thesis, TU Darmstadt / Mathematik.

Roy, A., Rumble, S. M., Stutsman, R., Levis, P., Mazières, D., and Zeldovich, N. 2011. Energy Management in Mobile Devices with the Cinder Operating System. In Proceedings of the Sixth Conference on Computer Systems. EuroSys '11. ACM, 139-152.

Ruiz, L. M., Valenciano, F., and Zarzuelo, J. M. 1996. The Least Square Prenucleolus and the Least Square Nucleolus. Two values for TU Games based on the excess vector. Int. J. Game Theory 25, 113-134.

Rumble, S. M., Stutsman, R., Levis, P., Mazières, D., And Zeldovich, N. 2009. Apprehending joule thieves with cinder. In Proceedings of the 1st ACM Workshop on Networking, Systems, and Applications for Mobile Handhelds. MobiHeld '09. ACM, New York, NY, USA, 49-54.

Ryffel, S. 2009. LEA ${ }^{2}$ P: The Linux Energy Attribution and Accounting Platform. M.S. thesis, Swiss Federal Institute of Technology.

Ryffel, S., Stathopoulos, T., McIntire, D., Kaiser, W., and Thiele, L. 2009. Accurate energy attribution and accounting for multi-core systems. Tech. rep., Center for Embedded Network Sensing, University of California. Sept.

Saha, M., Thakur, S., Singh, A., and Agarwal, Y. 2014. EnergyLens: Combining Smartphones with Electricity Meter for Accurate Activity Detection and User Annotation. In Proceedings of the 5th International Conference on Future Energy Systems. e-Energy '14. ACM, 289-300.

SChmeIdLer, D. 1969. The nucleolus of a characteristic function game. SIAM Journal of Applied Mathematics 17, 1163-1170.

Authors' preprint 
SEReno, M. 2012. Cooperative game theory framework for energy efficient policies in wireless networks. In Proceedings of the 3rd International Conference on Future Energy Systems: Where Energy, Computing and Communication Meet. e-Energy '12. ACM, 17:1-17:9.

Shapley, L. S. Cores of convex games. International Journal of Game Theory 1, 1, 11-26.

Shapley, L. S. 1953. A value for n-person games. Contributions to the theory of games 2, 307-317.

Sieber, A. And Nolte, J. 2013. Online device-level energy accounting for wireless sensor nodes. In Proceedings of the 10th European Conference on Wireless Sensor Networks. EWSN'13. Springer-Verlag, 149-164.

Thakur, S., Saha, M., Singh, A., And Agarwal, Y. 2014. Wattshare: Detailed energy apportionment in shared living spaces within commercial buildings. In Proceedings of the 1st ACM Conference on Embedded Systems for Energy-Efficient Buildings. BuildSys '14. ACM, 30-39.

TsaO, Y.-T., Huang, C.-C., And Hsu, J. Y.-J. 2014. Demand-based apportionment on electricity payment of hvac systems. In Proceedings of the 2014 International Conference on Autonomous Agents and Multi-agent Systems. AAMAS '14. International Foundation for Autonomous Agents and Multiagent Systems, 1601-1602.

Vallina-Rodriguez, N. And Crowcroft, J. 2011. Erdos: Achieving energy savings in mobile os. In Proceedings of the Sixth International Workshop on MobiArch. MobiArch '11. ACM, New York, NY, USA, 37-42.

Vergara, E. J., Andersson, S., And Nadjm-Tehrani, S. 2014a. When mice consume like elephants: Instant messaging applications. In Proceedings of the 5th International Conference on Future Energy Systems. e-Energy '14. ACM, 97-107.

Vergara, E. J., Nadjm-Tehrani, S., and Prihodko, M. Elsevier. 2014b. EnergyBox: Disclosing the wireless transmission energy cost for mobile devices. Sustainable Computing: Informatics and Systems 4, 2, 118-135.

Zeng, H., Ellis, C. S., Lebeck, A. R., And Vahdat, A. 2002a. ECOSystem: Managing Energy As a First Class Operating System Resource. SIGOPS Oper. Syst. Rev. 36, 5, 123-132.

Zeng, H., Ellis, C. S., Lebeck, A. R., And Vahdat, A. 2002b. Ecosystem: Managing energy as a first class operating system resource. SIGARCH Comput. Archit. News 30, 5, 123-132.

Zeng, H., Ellis, C. S., Lebeck, A. R., and Vahdat, A. 2003. Currentcy: A unifying abstraction for expressing energy management policies. In Proceedings of the Annual Conference on USENIX Annual Technical Conference. ATEC '03. USENIX Association, 4-4

Zhang, L., Tiwana, B., Qian, Z., Wang, Z., Dick, R. P., MaO, Z. M., And Yang, L. 2010. Accurate online power estimation and automatic battery behavior based power model generation for smartphones. In Proceedings of the Eighth IEEE/ACM/IFIP International Conference on Hardware/software Codesign and System Synthesis. CODES/ISSS '10. ACM, $105-114$.

Zhong, L. AND JHA, N. K. 2003. Graphical user interface energy characterization for handheld computers. In Proceedings of the 2003 International Conference on Compilers, Architecture and Synthesis for Embedded Systems. CASES '03. ACM, $232-242$.

Received September 2015; revised Month Year; accepted Month Year 


\title{
Online Appendix to: \\ Fairness and Incentive Considerations in Energy Apportionment Policies
}

\author{
EKHIOTZ JON VERGARA, SIMIN NADJM-TEHRANI and MIKAEL ASPLUND, \\ Linköping University
}

This appendix provides our proofs regarding the symmetry, dummy player, individual rationality, coalitional rationality and entity desirability properties shown in Table II. We also provide a reference to the properties that we do not analyse in this appendix.

Table III provides the reference to the works where the other properties are studied. The table also refers to the theorems in this appendix using a letter to specify the section and the theorem number. With the exception of entity desirability, the properties of the game-theoretical policies (Policies 5,6 and 7) are well known results in the game theory community, and thus we provide some references as examples. Note that the references of the coalitional rationality property refer to the individual rationality theorems since a policy does not satisfy coalitional rationality if it does not satisfy individual rationality.

Table III. : Reference to the fairness properties results for each policy.

\begin{tabular}{|c|c|c|c|c|c|}
\hline Nr. & $\mathbf{S}$ & DP & IR & CR & ED \\
\hline 1 & [Brink 2007] & $\begin{array}{l}\text { [González-Díaz and } \\
\text { Sánchez-Rodríguez } \\
\text { 2014] }\end{array}$ & $\begin{array}{l}\text { GGonzález-Díaz and } \\
\text { Sánchez-Rodríguez } \\
2014]\end{array}$ & Not IR & E.1 \\
\hline 2 & A.1 & B.1 & C.1 & C.1 & E.2 \\
\hline 3 & A.2 & B.2 & C.2 & C.2 & E.3 \\
\hline 4 & [Brink 2007] & $\begin{array}{l}\text { [González-Díaz and } \\
\text { Sánchez-Rodríguez } \\
2014]\end{array}$ & C. 3 & D. 1 & E.4 \\
\hline 5 & [Shapley 1953] & [Shapley 1953] & [McCain 2013, p. 151] & [Lemaire 1984, sec. 5] & [Rosenbusch 2011, p. 42] \\
\hline 6 & [Schmeidler 1969] & [Schmeidler 1969] & [Schmeidler 1969] & [Schmeidler 1969] & [Rosenbusch 2011, p. 151] \\
\hline 7 & [Ruiz et al. 1996] & [Ruiz et al. 1996] & [Ruiz et al. 1996] & [Ruiz et al. 1996] & [Ruiz et al. 1996] \\
\hline 8 & A. 3 & B. 3 & C. 4 & C. 4 & E. 5 \\
\hline 9 & A.4 & B.4 & C.5 & C.5 & E.6 \\
\hline 10 & A.5 & B.5 & C. 6 & C. 6 & E.7 \\
\hline
\end{tabular}

S: Symmetry. DP: Dummy player. IR: Individual rationality.

CR: Coalitional rationality. ED: Entity desirability.

\section{A. SYMMETRY}

This section presents the proofs regarding symmetry: Policies 2 and 3 satisfy the property, whereas Policy 8 , 9 and 10 do not satisfy it. The symmetry property presented in section 5.1 is the following: if $E(S \cup\{i\})=$ $E(S \cup\{j\})$ for all $S \subseteq N \backslash\{i, j\}$, then $\pi_{i}=\pi_{j}$.

Theorem A.1. Policy 2 (Proportional to isolation) always satisfies symmetry.

(C) 2016 ACM 0000-0000/2016/11-ART2 $\$ 15.00$

DOI 10.1145/2970816 http://doi.acm.org/10.1145/2970816 
Proof. In order to prove symmetry will assume $E(S \cup\{i\})=E(S \cup\{j\})$ for all $S \subseteq N \backslash\{i, j\}$, and prove that $\pi_{i}=\pi_{j}$ for Policy 2. From Equation 4 the apportionment for entities $i$ and $j$ is the following:

$$
\begin{aligned}
& \pi_{i}=E(\{i\}) \frac{E(N)}{\sum_{k \in N} E(\{k\})} \\
& \pi_{j}=E(\{j\}) \frac{E(N)}{\sum_{k \in N} E(\{k\})}
\end{aligned}
$$

It follows from the assumption that $E(\{i\})=E(\{j\})$ for $S=\emptyset$. Hence, from Equation 4 and $E(\{i\})=$ $E(\{j\})$ we have that:

$$
\pi_{i}=E(\{i\}) \frac{E(N)}{\sum_{k \in N} E(\{k\})}=E(\{j\}) \frac{E(N)}{\sum_{k \in N} E(\{k\})}=\pi_{j}
$$

Policy 2 always satisfies symmetry.

TheOrem A.2. Policy 3 (Marginal contribution) always satisfies symmetry.

Proof. In the proof we will assume $E(S \cup\{i\})=E(S \cup\{j\})$ for all $S \subseteq N \backslash\{i, j\}$, and prove $\pi_{i}=\pi_{j}$ for Policy 3.

We know that $E(S \cup\{i\})=E(N \backslash\{j\})$ and $E(S \cup\{j\})=E(N \backslash\{i\})$ for $S=N \backslash\{i, j\}$. Thus, it follow from the assumption that $E(N \backslash\{i\})=E(N \backslash\{j\})$.

From Equation 5 and $E(N \backslash\{i\})=E(N \backslash\{j\})$ we have the following:

$$
\begin{aligned}
& \pi_{i}=E(N \backslash\{i\}) \frac{E(N)}{\sum_{k \in N}[E(N)-E(N \backslash\{k\})]} \\
= & E(N \backslash\{j\}) \frac{E(N)}{\sum_{k \in N}[E(N)-E(N \backslash\{k\})]}=\pi_{j}
\end{aligned}
$$

Policy 3 satisfies symmetry.

Since activity and energy are not necessarily correlated we can easily find a counterexample for the surrogate-based policies.

Theorem A.3. Policy 8 (Proportional to activity) does not always satisfy symmetry.

Proof. A counterexample is enough to prove the theorem. We show that $E(S \cup\{i\})=E(S \cup\{j\})$ for all $S \subseteq N \backslash\{i, j\}$ holds in the counterexample and prove that $\pi_{i} \neq \pi_{j}$ for Policy 8 .

Table IV shows a system $N=\{1,2\}$ together with the energy consumption and activity function $a(S)$ for all possible configurations of this system.

Table IV. : Two entity game with symmetric entities.

\begin{tabular}{|c||c|c|c|}
\hline$S$ & $\{1\}$ & $\{2\}$ & $\{1,2\}$ \\
\hline$E(S)$ & 2 & 2 & 3 \\
\hline \hline$a(S)$ & 4 & 5 & 7 \\
\hline
\end{tabular}

Since $E(\{1\})=E(\{2\})$ for $S=\emptyset$ the antecedent for the symmetry requirement holds.

From Equation 10 and the values in Table IV the apportionment for entities 1 and 2 is computed: $\pi=$ $(1.33,1.66)$. Since $\pi_{1} \neq \pi_{2}$, Policy 8 does not satisfy symmetry.

Theorem A.4. Policy 9 (Time slicing) does not always satisfy symmetry.

Proof. A counterexample is enough to prove the theorem. We show that $E(S \cup\{i\})=E(S \cup\{j\})$ for all $S \subseteq N \backslash\{i, j\}$ holds in the counterexample and prove that $\pi_{i} \neq \pi_{j}$ for Policy 9 . 
Table IV and Figure 4 show the counterexample. Table IV shows a system $N=\{1,2\}$ together with the energy consumption and activity function $a(S)$ for all possible configurations of this system. Figure 4 shows the placement of the activities in time for the period $\left.T=\left[t_{1}, t_{5}\right]: s_{1}=\left[\left(t_{1}, 1\right),\left(t_{3}, 0\right)\right], s_{2}=\left[\left(t_{2}, 1\right),\left(t_{4}, 0\right)\right)\right]$, where $t_{1}=0, t_{2}=2, t_{3}=4, t_{4}=7$ and $t_{5}=10$.

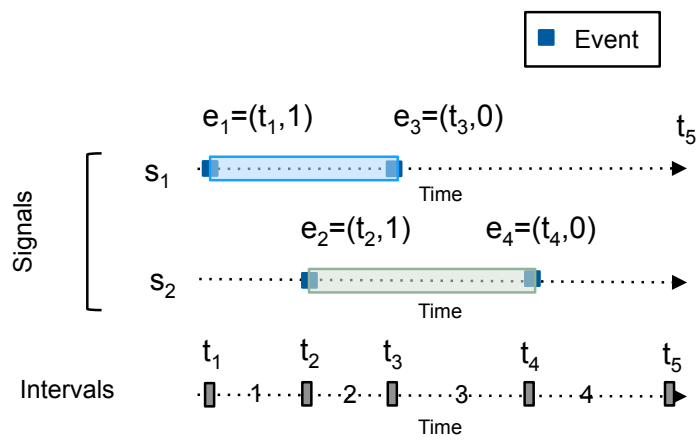

Fig. 4: Counterexample used in the proofs for symmetry for Policies 9 and 10.

Since $E(\{1\})=E(\{2\})$ for $S=\emptyset$ the antecedent for the symmetry requirement obviously holds. From Equation 11 we have that:

$$
\begin{gathered}
\pi_{1}=\frac{1}{10}\left[2+\frac{1}{2}(4-2)+0+\frac{1}{2}(10-7)\right] E(N)=1.35 \\
\pi_{2}=\frac{1}{10}\left[0+\frac{1}{2}(4-2)+(7-4)+\frac{1}{2}(10-7)\right] E(N)=1.65
\end{gathered}
$$

Hence, $\pi_{1} \neq \pi_{2}$. Policy 9 does not satisfy symmetry.

TheOrem A.5. Policy 10 (Last active) does not always satisfy symmetry.

Proof. A counterexample is enough to prove the theorem. We show that $E(S \cup\{i\})=E(S \cup\{j\})$ for all $S \subseteq N \backslash\{i, j\}$ holds for the counterexample and prove that $\pi_{i} \neq \pi_{j}$ for Policy 10 .

Table IV shows a system $N=\{1,2\}$ together with the energy consumption and activity function $a(S)$ for all possible configurations of this system. Figure 4 shows an illustration of the counterexample for the period $\left.T=\left[t_{1}, t_{5}\right]: s_{1}=\left[\left(t_{1}, 1\right),\left(t_{3}, 0\right)\right], s_{2}=\left[\left(t_{2}, 1\right),\left(t_{4}, 0\right)\right)\right]$, where $t_{1}=0, t_{2}=2, t_{3}=4, t_{4}=7$ and $t_{5}=10$. There are four intervals and all entities are inactive in the last interval, i.e., $\sum_{i \in N} a(i, 4)=0$. Entity 2 is the last active entity for interval $4: l(2,4)=1$. For simplicity we assume $E(N, j)=\frac{E(N)}{|J|}$ for the four intervals $j \in J$ $(|J|=4)$.

Since $E(\{1\})=E(\{2\})$ for $S=\emptyset$ the antecedent for the symmetry property is satisfied. The apportionment for entities 1 and 2 is computed from Equation 12:

$$
\begin{gathered}
\pi_{1}=\frac{E(N)}{|J|}+\frac{E(N)}{2|J|}+0+0=\frac{3 E(N)}{2|J|}=1.125 \\
\pi_{2}=0+\frac{E(N)}{2|J|}+\frac{E(N)}{|J|}+\frac{E(N)}{|J|}=\frac{5 E(N)}{2|J|}=1.875
\end{gathered}
$$

Hence, $\pi_{1} \neq \pi_{2}$. Policy 10 does not satisfy symmetry.

\section{B. DUMMY PLAYER}

In this section we provide the proofs for the dummy player property. Policies 2, 3, 8, 9 and 10 do not satisfy the dummy player property. The dummy player property presented in section 5.1 is the following: if $E(S \cup\{i\})-E(S)=E(\{i\})$ for all $S \subseteq N \backslash\{i\}$, then $\pi_{i}=E(\{i\})$. 
Theorem B.1. Policy 2 (Proportional to isolation) does not always satisfy dummy player.

Proof. It is enough to provide a counterexample to prove the theorem. We show that $E(S \cup\{i\})-E(S)=$ $E(\{i\})$ for all $S \subseteq N \backslash\{i\}$ holds in the counterexample and show that $\pi_{i} \neq E(\{i\})$ for Policy 2 .

Table $\mathrm{V}$ shows a system $N=\{1,2,3\}$ together with the energy consumption for all possible configurations of this system.

Table V. : A game where entity 2 is a dummy player.

\begin{tabular}{|c||c|c|c|c|c|c|c|}
\hline$S$ & $\{1\}$ & $\{2\}$ & $\{3\}$ & $\{1,2\}$ & $\{1,3\}$ & $\{2,3\}$ & $\{1,2,3\}$ \\
\hline$E(S)$ & 4 & 2 & 3 & 6 & 5 & 5 & 7 \\
\hline
\end{tabular}

Entity 2 satisfies the antecedent for the dummy player property:

$$
\begin{gathered}
E(\{1,2,3\})-E(\{1,3\})=E(\{2\}) \\
E(\{1,2\})-E(\{1\})=E(\{2\}) \\
E(\{2,3\})-E(\{3\})=E(\{2\}) \\
E(\{2\})-E(\emptyset)=E(\{2\})
\end{gathered}
$$

From Equation 4 and the values in Table V, the apportionment for the three entities is computed: $\pi=$ $(3.11,1.55,2.33)$. Clearly $\pi_{2} \neq E(\{2\})$ and thus Policy 2 does not satisfy the dummy player property.

The normalisation factor of Equation 4 is the main reason for not satisfying dummy player for Policy 2.

Theorem B.2. Policy 3 (Marginal contribution) does not always satisfy dummy player.

Proof. It is enough to provide a counterexample to prove the theorem. We show that $E(S \cup\{i\})-E(S)=$ $E(\{i\})$ for all $S \subseteq N \backslash\{i\}$ holds in the counterexample and prove that $\pi_{i} \neq E(\{i\})$ for Policy 3.

We employ the same counterexample as in the previous proof: Table $\mathrm{V}$ shows a system $N=\{1,2,3\}$ together with the energy consumption for all possible configurations of this system. Entity 2 satisfies the antecedent for the dummy player property.

From Equation 5 and the values in Table $\mathrm{V}$ the apportionment for the three entities is computed: $\pi=$ $(2.8,1.4,2.8)$. Clearly $\pi_{2} \neq E(\{2\})$ and thus Policy 3 does not satisfy the dummy player property.

Similarly to Policy 2, the normalisation factor of Equation 5 is the main reason for not satisfying dummy player for Policy 3.

Theorem B.3. Policy 8 (Proportional to activity) does not always satisfy dummy player.

Proof. It is enough to provide a counterexample to prove the theorem. We show that $E(S \cup\{i\})-E(S)=$ $E(\{i\})$ for all $S \subseteq N \backslash\{i\}$ holds in the counterexample and show that $\pi_{i} \neq E(\{i\})$ for Policy 8.

Table $\mathrm{V}$ shows a system $N=\{1,2,3\}$ together with the energy consumption for all possible configurations of the system. The values for the activity function are shown in Table VI which are the same as the $E(S)$ values.

Table VI. : Activity function values for the counterexample.

\begin{tabular}{|c||c|c|c|c|c|c|c|}
\hline$S$ & $\{1\}$ & $\{2\}$ & $\{3\}$ & $\{1,2\}$ & $\{1,3\}$ & $\{2,3\}$ & $\{1,2,3\}$ \\
\hline$a(S)$ & 4 & 2 & 3 & 6 & 5 & 5 & 7 \\
\hline
\end{tabular}

Authors' preprint 
Entity 2 satisfies the antecedent for the dummy player property from the values of Table $\mathrm{V}$ as shown in the Theorem B.1.

From Equation 10 the apportionment for the entities is computed: $\pi=(3.11,1.55,2.33)$. Obviously $\pi_{2} \neq$ $E(\{2\})$. Hence, Policy 8 does not satisfy the dummy player property.

Similarly to the Policies 2 and 3, the normalisation factor is the main reason for not satisfying the dummy player.

Theorem B.4. Policy 9 (Time slicing) does not always satisfy dummy player.

Proof. It is enough to provide a counterexample to prove the theorem. We show that $E(S \cup\{i\})-E(S)=$ $E(\{i\})$ for all $S \subseteq N \backslash\{i\}$ holds in the counterexample and show that $\pi_{i} \neq E(\{i\})$ for Policy 9.

Tables V and VI show a system $N=\{1,2,3\}$ and the energy consumption and activity function for every configuration of the system. An illustration of the counterexample is shown in Figure 5 for a period $T=\left[t_{1}, t_{7}\right]: s_{1}=\left[\left(t_{1}, 1\right),\left(t_{3}, 0\right)\right], s_{2}=\left[\left(t_{5}, 1\right),\left(t_{6}, 0\right)\right], s_{3}=\left[\left(t_{2}, 1\right),\left(t_{4}, 0\right)\right]$, where $t_{1}=0, t_{2}=2, t_{3}=4$, $t_{4}=5, t_{5}=6, t_{6}=8$ and $t_{7}=10$. There are 6 intervals $(|J|=6)$ where there are no active entities for intervals 4 and 6, i.e., $\sum_{i \in N} a(i, 4)=0$ and $\sum_{i \in N} a(i, 6)=0$.

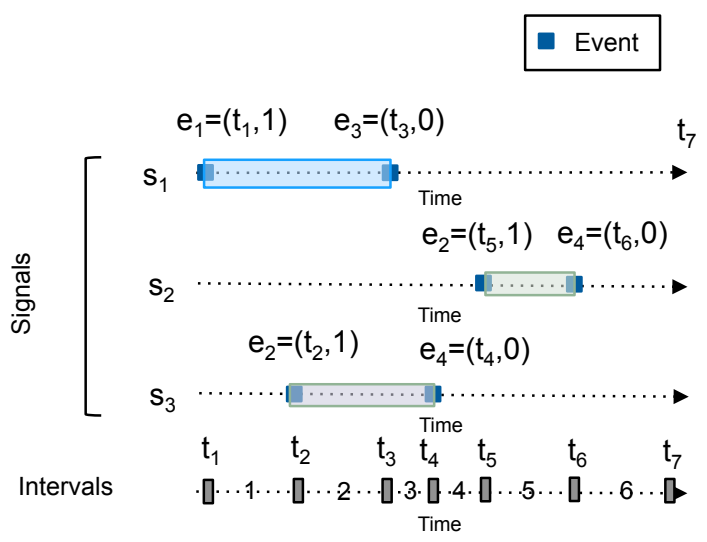

Fig. 5: Illustration of the counterexample used in the proofs for dummy player for Policies 9 and 10.

Entity 2 satisfies the antecedent for the dummy player property from the values of Table $\mathrm{V}$ as shown in the Theorem B.1.

From Equation 11 the apportionment for the entities is computed:

$$
\begin{aligned}
& \pi_{1}=\frac{1}{10}\left[2+\frac{2}{2}+0+\frac{1}{3}+0+\frac{2}{3}\right] E(N)=2.8 \\
& \pi_{2}=\frac{1}{10}\left[0+0+0+\frac{1}{3}+2+\frac{2}{3}\right] E(N)=2.1 \\
& \pi_{3}=\frac{1}{10}\left[0+\frac{2}{2}+1+\frac{1}{3}+0+\frac{2}{3}\right] E(N)=2.1
\end{aligned}
$$

Clearly $\pi_{2} \neq E(\{2\})$, and thus Policy 9 does not satisfy the dummy player property.

Theorem B.5. Policy 10 (Last active) does not always satisfy dummy player.

Proof. It is enough to provide a counterexample to prove the theorem. We show that $E(S \cup\{i\})-E(S)=$ $E(\{i\})$ for all $S \subseteq N \backslash\{i\}$ holds in the counterexample and show that $\pi_{i} \neq E(\{i\})$ for Policy 10.

Tables $\mathrm{V}$ and VI show a system $N=\{1,2,3\}$ and the energy consumption and activity function for every configuration of the system. An illustration of the counterexample is shown in Figure 5 for a period $T=\left[t_{1}, t_{7}\right]: s_{1}=\left[\left(t_{1}, 1\right),\left(t_{3}, 0\right)\right], s_{2}=\left[\left(t_{5}, 1\right),\left(t_{6}, 0\right)\right], s_{3}=\left[\left(t_{2}, 1\right),\left(t_{4}, 0\right)\right]$, where $t_{1}=0, t_{2}=2, t_{3}=4$, 
$t_{4}=5, t_{5}=6, t_{6}=8$ and $t_{7}=10$. There are 6 intervals $(|J|=6)$ where there are no active entities for intervals 4 and 6, i.e., $\sum_{i \in N} a(i, 4)=0$ and $\sum_{i \in N} a(i, 6)=0$. For these intervals we have that $l(3,4)=1$ and $l(2,6)=1$, i.e., entity 3 is the last active entity for interval 4 and entity 2 is the last active entity for interval 6 respectively. For simplicity we assume $E(N, j)=\frac{E(N)}{|J|}$ for the four intervals $j \in J(|J|=6)$.

Entity 2 satisfies the antecedent for the dummy player property from the values of Table $\mathrm{V}$ as shown in the Theorem B.1.

The apportionment for entities 1, 2 and 3 is computed from Equation 12:

$$
\begin{gathered}
\pi_{1}=\frac{E(N)}{|J|}+\frac{E(N)}{2|J|}+0+0+0+0=\frac{3 E(N)}{2|J|}=1.75 \\
\pi_{2}=0+0+0+0+\frac{E(N)}{|J|}+\frac{E(N)}{|J|}=\frac{2 E(N)}{|J|}=2.33 \pi_{3}=0+\frac{E(N)}{2|J|}+\frac{E(N)}{|J|}+\frac{E(N)}{|J|}+0+0=\frac{5 E(N)}{2|J|}=2.92
\end{gathered}
$$

Obviously $\pi_{2} \neq E(\{2\})$ and thus Policy 10 does not satisfy the dummy player property.

Null player: Now we show that even though Policies 2, 3, 8 and 10 do not always satisfy dummy player, they satisfy a basic case of dummy player known as the null player property: if $E(S \cup\{i\})-E(S)=0$ for all $S \subseteq N \backslash\{i\}$, then $\pi_{i}=0$.

Theorem B.6. Policy 2 (Proportional to isolation) always satisfies null player.

Proof. In the proof we assume $E(S \cup\{i\})-E(S)=0$ for all $S \subseteq N \backslash\{i\}$ and show $\pi_{i}=0$.

It follows from the antecedent of the null player property that $E(\{\bar{i}\})=0$ for $S=\emptyset$. From Equation 4 and $E(\{i\})=0$ we have the following:

$$
\pi_{i}=\frac{E(\{i\})}{\sum_{j \in N} E(\{j\})} E(N)=\frac{0}{\sum_{j \in N} E(\{j\})} E(N)=0
$$

Thus, Policy 2 satisfies the null player property.

TheORem B.7. Policy 3 (Marginal contribution) always satisfies null player.

Proof. In the proof we assume $E(S \cup\{i\})-E(S)=0$ for all $S \subseteq N \backslash\{i\}$ and show $\pi_{i}=0$.

It follows from the antecedent of the null player property that $E(N)-E(N \backslash\{i\})=0$ for $S=N \backslash\{i\}$. From Equation 5 and $E(N)-E(N \backslash\{i\})=0$ we have the following:

$$
\begin{aligned}
& \pi_{i}=\frac{E(N)-E(N \backslash\{i\})}{\sum_{j \in N}[E(N)-E(N \backslash\{j\})]} E(N) \\
= & \frac{0}{\sum_{j \in N}[E(N)-E(N \backslash\{j\})]} E(N)=0
\end{aligned}
$$

Hence, Policy 3 satisfies the null player property.

The notion of null player was defined in terms of energy. When we discuss surrogate-based policies we should envisage a surrogate type of null player property. Intuitively, it makes sense that if an entity does not have a surrogate attribute in a scenario (e.g., it does not have any activity), it does not consume energy in isolation and vice versa. Hence, $a(i)=0$ iff $E(\{i\})=0$.

TheOREM B.8. Policy 8 (Proportional to activity) always satisfies null player.

Proof. In the proof we assume $E(S \cup\{i\})-E(S)=0$ for all $S \subseteq N \backslash\{i\}$ and show $\pi_{i}=0$.

It follows from the antecedent of the null player property that $E(\{i\})=0$ for $S=\emptyset$. Since $E(\{i\})=0$, $a(i)=0$. From Equation 10 and $a(i)=0$ we have the following:

$$
\pi_{i}=\frac{a(i)}{\sum_{j \in N} a(j)} E(N)=\frac{0}{\sum_{j \in N} a(j)} E(N)=0
$$

Hence, Policy 8 satisfies the null player property.

TheOREM B.9. Policy 9 (Time slicing) does not always satisfy null player. 
Proof. A counterexample is enough to prove the theorem. We show that $E(S \cup\{i\})-E(S)=0$ for all $S \subseteq N \backslash\{i\}$ holds in the counterexample and show $\pi_{i} \neq 0$.

Table VII and Figure 6 show the counterexample. Table VII shows a system $N=\{1,2\}$ and the energy consumption and activity function for all possible configurations of this system. Figure 6 shows the placement of the activities in time for the period $T=10: s_{1}=\left[\left(t_{1}, 1\right),\left(t_{2}, 0\right)\right]$ and $s_{2}=[]$ (no events), where $t_{1}=0$, $t_{2}=5$ and $t_{3}=10$. There are two intervals and in the last interval there is no active entity $\left(\sum_{i \in N} a(i, 2)=0\right)$.

Table VII. : A counterexample used to show that Policy 9 does not satisfy then null player property.

\begin{tabular}{|c||c|c|c|}
\hline$S$ & $\{1\}$ & $\{2\}$ & $\{12\}$ \\
\hline$E(S)$ & 3 & 0 & 3 \\
\hline \hline$a(S)$ & 5 & 0 & 5 \\
\hline
\end{tabular}

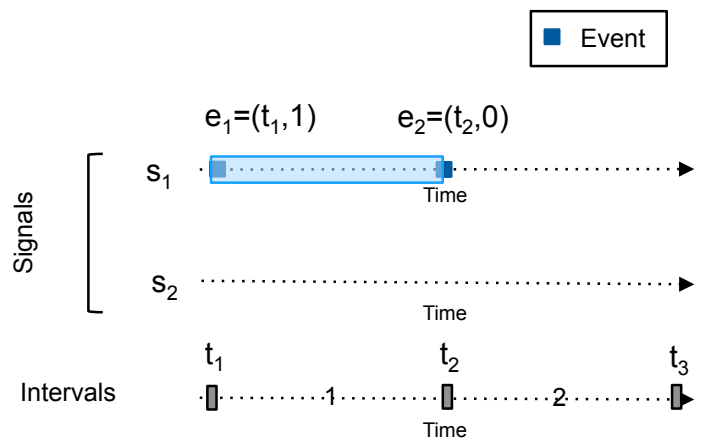

Fig. 6: Illustration of the counterexample used in the proof for null player for Policy 10.

Since $E(\{2\})-E(\{\emptyset\})=0, E(\{1,2\})-E(\{1\})=0$ and $a(2)=0$ from Table VII entity 2 satisfies the antecedent for the null player property.

From Equation 11 the apportionment is computed:

$$
\begin{aligned}
& \pi_{1}=\frac{1}{10}\left[5+\frac{5}{2}\right] E(N)=2.25 \\
& \pi_{2}=\frac{1}{10}\left[0+\frac{5}{2}\right] E(N)=0.75
\end{aligned}
$$

Clearly $\pi_{2} \neq 0$ and thus Policy 9 does not satisfy the null player property.

TheOrem B.10. Policy 10 (Last active) always satisfies null player.

Proof. In the proof we assume $E(S \cup\{i\})-E(S)=0$ for all $S \subseteq N \backslash\{i\}$ and show $\pi_{i} \neq 0$.

From $E(S \cup\{i\})-E(S)=0$ for all $S \subseteq N \backslash\{i\}$ we have that $E(\{i\})=0$ for $S=\emptyset$. Since $E(\{i\})=0$ $a(i)=0$. Thus, $a(i, j)=0$ for all $j$. From Equation 12 there are two cases for $g(i, j)$ :

(1) If $\sum_{i \in N} a(i, j)=0$, then $g(i, j)=l(i, j)$. Since $a(i, j)=0, l(i, j)=0$ for all $j$ (if an entity was not active, it cannot be the last active entity). Thus, $g(i, j)=0$.

(2) If $\sum_{i \in N} a(i, j) \neq 0$, then $g(i, j)=\frac{a(i, j)}{\sum_{k \in N} a(k, j)}$. Since $a(i, j)=0$ we have that $g(i, j)=0$.

Since $g(i, j)=0$ for all $j, \pi_{i}=0$. Hence, Policy 10 satisfies the null player property.

Note that Policy 10 does not satisfy dummy player due to the last active entity mechanism, whereas it satisfies null player since an entity that never is active cannot be the last active entity. 


\section{INDIVIDUAL RATIONALITY}

In this section we provide the proofs for individual rationality. Policy 2 and 4 always satisfy individual rationality. Policies 3, 8, 9 and 10 do not always satisfy individual rationality. Individual rationality states that if $I(E) \neq \emptyset$ then $\pi_{i} \leq E(\{i\}), \forall i \in N$. It is well known that $\sum_{i \in N} E(\{i\}) \geq E(N)$ iff $I(E) \neq \emptyset$.

Theorem C.1. Policy 2 (Proportional to isolation) always satisfies individual rationality.

Proof. In the proof we will assume $I(E) \neq \emptyset$ and show $\pi_{i} \leq E(\{i\}), \forall i \in N$.

It follows from the antecedent of individual rationality that $\sum_{i \in N} E(\{i\}) \geq E(N)$. Thus:

$$
\frac{E(N)}{\sum_{i \in N} E(\{i\})} \leq 1
$$

Consequently, from Equation 4 we have that:

$$
\begin{gathered}
\pi_{i}=E(\{i\}) \frac{E(N)}{\sum_{j \in N} E(\{j\})} \\
\pi_{i} \leq E(\{i\})
\end{gathered}
$$

Hence, Policy 2 satisfies individual rationality.

Theorem C.2. Policy 3 (Marginal contribution) does not always satisfy individual rationality.

Proof. A counterexample is enough to prove the theorem. We show that $I(E) \neq \emptyset$ holds in the counterexample and show $\pi_{i} \leq E(\{i\}), \forall i \in N$ is not satisfied for Policy 3 .

Table VIII shows a system $N=\{1,2,3\}$ and the energy consumption for all possible configurations of this system.

Table VIII. : An example where Policy 3 does not satisfy individual rationality.

\begin{tabular}{|c||c|c|c|c|c|c|c|}
\hline$S$ & $\{1\}$ & $\{2\}$ & $\{3\}$ & $\{1,2\}$ & $\{1,3\}$ & $\{2,3\}$ & $\{1,2,3\}$ \\
\hline$E(S)$ & 2 & 1 & 2 & 2 & 2 & 2 & 4 \\
\hline
\end{tabular}

$\sum_{i \in N} E(\{i\}) \geq E(N)$ is satisfied from the values in Table VIII $(2+1+2>4)$, and thus $I(E) \neq \emptyset$ holds for the counterexample.

From Equation 5 and the values in Table VIII the apportionment is computed: $\pi=(1.33,1.33,1.33)$. Clearly $\pi_{2}>E(\{2\})$, and thus Policy 3 does not satisfy individual rationality.

Since Policy 3 does not look at the energy consumption of the singletons, it fails to be individually rational. Instead, for the same game, Policy 2 results in $\pi=(1.6,0.8,1.6)$ which is individually rational.

Theorem C.3. Policy 4 (Isolation energy and remainder) always satisfies individual rationality.

Proof. In the proof we will assume $I(E) \neq \emptyset$ and show $\pi_{i} \leq E(\{i\}), \forall i \in N$.

It follows from the antecedent of individual rationality that $\sum_{i \in N} E(\{i\}) \geq E(N)$. Thus:

$$
E(N)-\sum_{i \in N} E(\{i\}) \leq 0
$$

Consequently, from Equation 6 we have that:

$$
\begin{gathered}
\pi_{i}=E(\{i\})+\frac{E(N)-\sum_{j \in N} E(\{j\})}{|N|} \\
\pi_{i} \leq E(\{i\})
\end{gathered}
$$

Authors' preprint 
Hence, Policy 4 satisfies individual rationality.

Theorem C.4. Policy 8 (Proportional to activity) does not always satisfy individual rationality.

Proof. A counterexample is enough to prove the theorem. We show that $I(E) \neq \emptyset$ holds in the counterexample and prove that the following is not satisfied $\pi_{i} \leq E(\{i\}), \forall i \in N$ for Policy 8 .

Table VIII shows a system $N=\{1,2,3\}$, the energy consumption and the activity function for all possible configurations of this system.

Table IX. : A counterexample used to show that Policies 8, 9 and 10 do not always satisfy individual rationality.

\begin{tabular}{|c||c|c|c|c|c|c|c|}
\hline$S$ & $\{1\}$ & $\{2\}$ & $\{3\}$ & $\{1,2\}$ & $\{1,3\}$ & $\{2,3\}$ & $\{1,2,3\}$ \\
\hline$E(S)$ & 2 & 1 & 2 & 2 & 2 & 2 & 4 \\
\hline \hline$a(S)$ & 4 & 3 & 4 & 7 & 6 & 6 & 8 \\
\hline
\end{tabular}

$\sum_{i \in N} E(\{i\}) \geq E(N)$ is satisfied from the values in Table IX $(2+1+2>4)$, and thus $I(E) \neq \emptyset$ holds for the counterexample.

From Equation 10 and the values in Table IX the apportionment for the three entities is computed: $\pi=(1.45,1.09,1.45)$. Obviously $\pi_{2}>E(\{2\})$. Policy 8 does not satisfy individual rationality.

Theorem C.5. Policy 9 (Time slicing) does not always satisfy individual rationality.

Proof. A counterexample is enough to prove the theorem. We show that $I(E) \neq \emptyset$ holds in the counter example and prove that $\pi_{i} \leq E(\{i\}), \forall i \in N$ is not satisfied for Policy 9 .

Table VIII and Figure 7 show the counterexample. Table VIII shows a system $N=\{1,2,3\}$, the energy consumption and the activity function for all possible configurations of this system. Figure 7 shows the placement of the activities in time for the period $T=10: s_{1}=\left[\left(t_{1}, 1\right),\left(t_{3}, 0\right)\right], s_{2}=\left[\left(t_{4}, 1\right),\left(t_{6}, 0\right)\right]$ and $s_{3}=\left[\left(t_{2}, 1\right),\left(t_{5}, 0\right)\right]$, where $t_{1}=0, t_{2}=2, t_{3}=4, t_{4}=5, t_{5}=6, t_{6}=8$ and $t_{7}=10$. There are 6 intervals and for the last interval there is no active entity $\left(\sum_{i \in N} a(i, 6)=0\right)$.

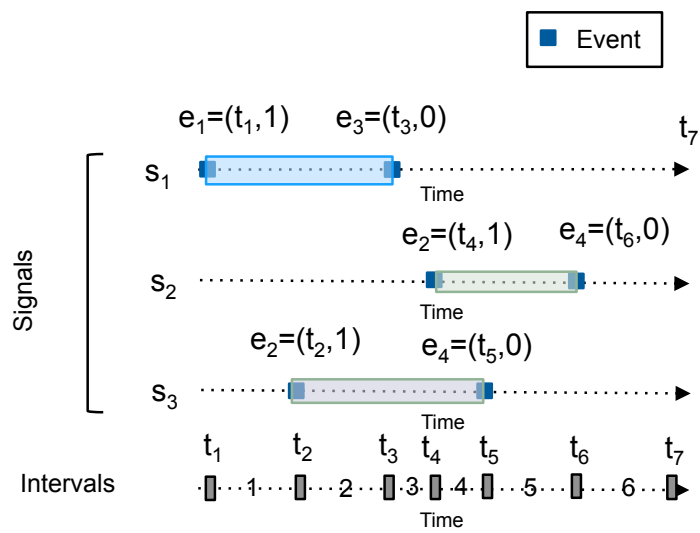

Fig. 7: Illustration of the counterexample used in the proofs for individual rationality for Policies 8,9 and 10.

$\sum_{i \in N} E(\{i\}) \geq E(N)$ is satisfied from the values in Table IX $(2+1+2>4)$, and thus $I(E) \neq \emptyset$ holds for the counterexample.

From Equation 11 the apportionment for the entities 1, 2 and 3 is computed: 


$$
\begin{aligned}
& \pi_{1}=\frac{1}{10}\left[2+\frac{2}{2}+0+0+0+\frac{2}{3}\right] E(N)=1.46 \\
& \pi_{2}=\frac{1}{10}\left[0+0+0+\frac{1}{2}+2+\frac{2}{3}\right] E(N)=1.26 \\
& \pi_{3}=\frac{1}{10}\left[0+\frac{2}{2}+1+\frac{1}{2}+0+\frac{2}{3}\right] E(N)=1.26
\end{aligned}
$$

Clearly $\pi_{2}>E(\{2\})$, and thus Policy 9 does not satisfy individual rationality.

Theorem C.6. Policy 10 (Last active) does not always satisfy individual rationality .

Proof. A counterexample is enough to prove the theorem. We show that $I(E) \neq \emptyset$ holds in the counter example and prove that $\pi_{i} \leq E(\{i\}), \forall i \in N$ is not satisfied for Policy 10 .

Table VIII and Figure 7 show the counterexample. Table VIII shows a system $N=\{1,2,3\}$, the energy consumption and the activity function for all possible configurations of this system. Figure 7 shows the placement of the activities in time for the period $T=10: s_{1}=\left[\left(t_{1}, 1\right),\left(t_{3}, 0\right)\right], s_{2}=\left[\left(t_{4}, 1\right),\left(t_{6}, 0\right)\right]$ and $s_{3}=\left[\left(t_{2}, 1\right),\left(t_{5}, 0\right)\right]$, where $t_{1}=0, t_{2}=2, t_{3}=4, t_{4}=5, t_{5}=6, t_{6}=8$ and $t_{7}=10$. There are 6 intervals and for the last interval there is no active entity $\left(\sum_{i \in N} a(i, 6)=0\right)$. Thus, $l(2,6)=1$. For simplicity we assume $E(N, j)=\frac{E(N)}{|J|}$ for the six intervals $j \in J(|J|=6)$.

$\sum_{i \in N} E(\{i\}) \geq E(N)$ is satisfied from the values in Table IX $(2+1+2>4)$, and thus $I(E) \neq \emptyset$ holds for the counterexample.

From Equation 12 the apportionment for the counterexample is computed:

$$
\begin{gathered}
\pi_{1}=\frac{E(N)}{|J|}+\frac{E(N)}{2|J|}+0+0+0+0=\frac{3 E(N)}{2|J|}=1 \\
\pi_{2}=0+0+0+\frac{E(N)}{2|J|}+\frac{E(N)}{|J|}+\frac{E(N)}{|J|}=\frac{5 E(N)}{2|J|}=1.66 \\
\pi_{3}=0+\frac{E(N)}{2|J|}+\frac{E(N)}{|J|}+\frac{E(N)}{2|J|}+0+0=\frac{2 E(N)}{|J|}=1.33
\end{gathered}
$$

Obviously $\pi_{2}>E(\{2\})$ and thus Policy 10 does not satisfy individual rationality.

\section{COALITIONAL RATIONALITY}

In this section we provide the proofs for the conditional rationality property which states that if $C(E) \neq \emptyset$ then $\sum_{i \in S} \pi_{i} \leq E(S) \forall S \subseteq N$. The property includes individual rationality when $|S|=1$, and thus Policies $1,3,8,9$ and 10 that do not satisfy individual rationality do not satisfy coalitional rationality either. In this section we provide the proof showing that Policy 4 does not satisfy coalitional rationality even though it satisfies individual rationality.

Theorem D.1. Policy 4 (Isolation energy and remainder) does not always satisfy coalitional rationality.

Proof. A counterexample is enough to prove the theorem. We show that $C(E) \neq \emptyset$ in the counterexample and show that $\sum_{i \in S} \pi_{i} \leq E(S) \forall S \subseteq N$ is not satisfied for Policy 4 .

Table $\mathrm{X}$ shows a system $N=\{1,2,3\}$ and the energy consumption for every possible system configuration.

Table X. : Counterexample to prove that Policy 4 does not satisfy coalitional rationality.

\begin{tabular}{|c||c|c|c|c|c|c|c|}
\hline$S$ & $\{1\}$ & $\{2\}$ & $\{3\}$ & $\{1,2\}$ & $\{1,3\}$ & $\{2,3\}$ & $\{1,2,3\}$ \\
\hline$E(S)$ & 3 & 3 & 4 & 4.5 & 5.5 & 6 & 8 \\
\hline
\end{tabular}

An individually rational apportionment satisfies the following inequalities:

$$
\begin{aligned}
& \pi_{1} \leq E(\{1\}) \\
& \pi_{2} \leq E(\{2\}) \\
& \pi_{3} \leq E(\{3\})
\end{aligned}
$$


Additionally, a coalitionally rational apportionment satisfies also the following inequalities:

$$
\begin{gathered}
\pi_{1}+\pi_{2} \leq E(\{1,2\}) \\
\pi_{1}+\pi_{3} \leq E(\{1,3\}) \\
\pi_{2}+\pi_{3} \leq E(\{2,3\}) \\
\pi_{1}+\pi_{2}+\pi_{3} \leq E(\{1,2,3\})
\end{gathered}
$$

The counterexample in Table $\mathrm{X}$ satisfies $C(E) \neq \emptyset$ since the inequality system has at least an answer (e.g., $\pi=(2,2.5,3.5))$.

From Equation 6 the apportionment for entities 1, 2 and 3 is computed: $\pi=(2.33,2.33,3.33) . \pi$ is individually rational since $\pi_{1}<E(\{1\}), \pi_{2}<E(\{2\})$ and $\pi_{3}<E(\{3\})$. For coalitional rationality we have the following:

$$
\begin{gathered}
2.33+2.33>E(\{1,2\}) \\
2.33+3.33>E(\{1,3\}) \\
2.33+3.33<E(\{2,3\}) \\
2.33+2.33+3.33<E(\{1,2,3\})
\end{gathered}
$$

Clearly $\pi_{1}+\pi_{2}>E(\{1,2\})$ and $\pi_{2}+\pi_{3}>E(\{1,3\})$, and thus Policy 4 does not satisfy coalitional rationality.

The main reason for Policy 4 to not satisfy coalitional rationality is that Equation 6 only consider the value of the singleton coalitions to apportion the energy consumption of the grand coalition.

\section{E. ENTITY DESIRABILITY}

In this section we provide the proofs showing that Policies 1, 2, 3 and 4 satisfy entity desirability and Policies 8,9 and 10 do not satisfy entity desirability.

Entity desirability states that $i \succeq j \Longrightarrow \pi_{i} \leq \pi_{j}$, where $i \succeq j$ is defined as $E(S \cup\{i\}) \leq E(S \cup\{j\}) \forall S \subseteq$ $N \backslash\{i, j\}$.

Theorem E.1. Policy 1 always satisfies entity desirability.

Proof. In order to prove the theorem we assume $i \succeq j$ and show $\pi_{i} \leq \pi_{j}$ for Equation 3 .

From Equation 3 we have that $\pi_{i}=\pi_{j}$ for all $i, j \in N$. Hence, Policy 1 trivially satisfies entity desirability.

Theorem E.2. Policy 2 (Proportional to isolation) always satisfies entity desirability.

Proof. In order to prove it we assume $i \succeq j$ and show $\pi_{i} \leq \pi_{j}$ for Equation 4 .

It follows from the antecedent of entity desirability that $E(\{i\}) \leq E(\{j\})$ for $S=\emptyset$. From Equation 4 and $E(\{i\}) \leq E(\{j\})$ we have the following for $i$ and $j$ :

$$
\begin{aligned}
E(\{i\}) \frac{E(N)}{\sum_{k \in N} E(\{k\})} & \leq E(\{j\}) \frac{E(N)}{\sum_{k \in N} E(\{k\})} \\
\pi_{i} & \leq \pi_{j}
\end{aligned}
$$

Hence, Policy 2 satisfies entity desirability.

Theorem E.3. Policy 3 (Marginal contribution) always satisfies entity desirability.

Proof. In order to prove the theorem we assume $i \succeq j$ and show $\pi_{i} \leq \pi_{j}$ for Equation 5 .

We know that $E(S \cup\{i\})=E(N \backslash\{j\})$ and $E(S \cup\{j\})=E(N \backslash\{i\})$ for $S=N \backslash\{i, j\}$. Thus, it follows from the assumption that $E(N \backslash\{j\}) \leq E(N \backslash\{i\})$. Hence, $E(N)-E(N \backslash\{j\}) \geq E(N)-E(N \backslash\{i\})$.

From Equation 5 and $E(N)-E(N \backslash\{i\}) \leq E(N)-E(N \backslash\{j\})$ we have the following for $i$ and $j$ : 


$$
\begin{aligned}
\frac{E(N)-E(N \backslash\{i\})}{\sum_{k \in N}[E(N)-E(N \backslash\{k\})]} E(N) & \leq \frac{E(N)-E(N \backslash\{j\})}{\sum_{k \in N}[E(N)-E(N \backslash\{k\})]} E(N) \\
\pi_{i} & \leq \pi_{j}
\end{aligned}
$$

Thus, Policy 3 satisfies entity desirability.

TheOrem E.4. Policy 4 always satisfies entity desirability.

Proof. In order to prove the theorem we assume $i \succeq j$ and prove that for Equation 6 we have $\pi_{i} \leq \pi_{j}$.

From $i \succeq j$ it follows that $E(\{i\}) \leq E(\{j\})$ for $S=\emptyset$. From Equation 6 and $E(\{i\}) \leq E(\{j\})$ we have that:

$$
\begin{aligned}
E(\{i\})+\frac{E(N)-\sum_{k \in N} E(\{k\})}{|N|} & \leq E(\{j\})+\frac{E(N)-\sum_{k \in N} E(\{k\})}{|N|} \\
\pi_{i} & \leq \pi_{j}
\end{aligned}
$$

Hence, Policy 4 satisfies entity desirability.

Theorem E.5. Policy 8 (Proportional to activity) does not always satisfy entity desirability.

Proof. It is enough to provide a counterexample to prove the theorem. We show $i \succeq j$ in the counterexample and prove $\pi_{i}>\pi_{j}$.

Table XI. : A counterexample used to show that Policies 8, 9 and 10 do not satisfy entity desirability.

\begin{tabular}{|c||c|c|c|}
\hline$S$ & $\{1\}$ & $\{2\}$ & $\{12\}$ \\
\hline$E(S)$ & 4 & 5 & 3 \\
\hline \hline$a(S)$ & 4 & 3 & 6 \\
\hline
\end{tabular}

Table XI shows a system $N=\{1,2\}$, the energy consumption and the activity function for every possible configuration of this system.

We know that $S=N \backslash\{1,2\}=\emptyset$. Thus, $1 \succeq 2$ is $E(S \cup\{1\}) \leq E(S \cup\{2\})$ for $S=\emptyset$, i.e., $E(\{1\}) \leq E(\{2\})$. This inequality holds for the values in Table XI. Thus, $1 \succeq 2$ holds in the counterexample.

From Equation 10 and the values in Table XI the apportionment for the entities is computed: $\pi=$ $(1.72,1.28)$. Clearly $\pi_{1}>\pi_{2}$, and thus Policy 8 does not satisfy entity desirability.

TheORem E.6. Policy 9 (Time slicing) does not always satisfy entity desirability.

Proof. It is enough to provide a counterexample to prove the theorem. We show $i \succeq j$ in the counterexample and prove $\pi_{i}>\pi_{j}$ for Policy 9 .

Table XI and Figure 8 show the counterexample. Table XI shows a system $N=\{1,2\}$, the energy consumption and the activity function for every possible configuration of this system. Figure 8 shows the placement of the activities in time for the period $T=10: s_{1}=\left[\left(t_{2}, 1\right),\left(t_{4}, 0\right)\right]$ and $s_{2}=\left[\left(t_{1}, 1\right),\left(t_{3}, 0\right)\right]$ where $t_{1}=0$, $t_{2}=2, t_{3}=3, t_{4}=6$ and $t_{5}=10$. There are four intervals and there is no active entity in the last interval $\left(\sum_{i \in N} a(i, 4)=0\right)$.

We know that $S=N \backslash\{1,2\}=\emptyset$. Thus, $1 \succeq 2$ is $E(S \cup\{1\}) \leq E(S \cup\{2\})$ for $S=\emptyset$, i.e., $E(\{1\}) \leq E(\{2\})$. This inequality holds for the values in Table XI. Thus, $1 \succeq 2$ holds in the counterexample.

From Equation 11 the apportionment for entities 1 and 2 is computed:

$$
\begin{aligned}
& \pi_{1}=\frac{1}{10}\left[0+\frac{1}{2}+3+\frac{4}{2}\right] E(N)=1.65 \\
& \pi_{2}=\frac{1}{10}\left[2+\frac{1}{2}+0+\frac{4}{2}\right] E(N)=1.35
\end{aligned}
$$

Obviously $\pi_{1}>\pi_{2}$, and thus Policy 9 does not satisfy entity desirability. 


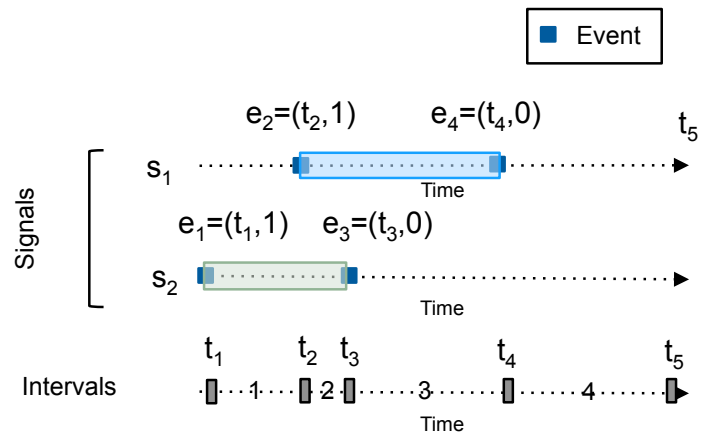

Fig. 8: Counterexample used to show that Policies 8,9 and 10 do not satisfy entity desirability.

TheOrem E.7. Policy 10 (Last active) does not always satisfy entity desirability.

Proof. It is enough to provide a counterexample to prove the theorem. We show $i \succeq j$ in the counterexample and prove $\pi_{i}>\pi_{j}$ for Policy 10 .

Table XI and Figure 8 show the counterexample. Table XI shows a system $N=\{1,2\}$, the energy consumption and the activity function for every possible configuration of this system. Figure 8 shows the placement of the activities in time for the period $T=10: s_{1}=\left[\left(t_{2}, 1\right),\left(t_{4}, 0\right)\right]$ and $s_{2}=\left[\left(t_{1}, 1\right),\left(t_{3}, 0\right)\right]$ where $t_{1}=0$, $t_{2}=2, t_{3}=3, t_{4}=6$ and $t_{5}=10$. There are four intervals and there is no active entity in the last interval $\left(\sum_{i \in N} a(i, 4)=0\right) \cdot l(1,4)=1$ since entity 1 is the last active entity for interval 4 . For simplicity we assume $E(N, j)=\frac{E(N)}{|J|}$ for the four intervals $j \in J(|J|=4)$.

We know that $S=N \backslash\{1,2\}=\emptyset$. Thus, $1 \succeq 2$ is $E(S \cup\{1\}) \leq E(S \cup\{2\})$ for $S=\emptyset$, i.e., $E(\{1\}) \leq E(\{2\})$. This inequality holds for the values in Table XI. Thus, $1 \succeq 2$ holds in the counterexample.

From Equation 12 the apportionment for the counterexample is computed:

$$
\begin{gathered}
\pi_{1}=0+\frac{E(N)}{2|J|}+\frac{E(N)}{|J|}+\frac{E(N)}{|J|}=\frac{5 E(N)}{2|J|}=1.875 \\
\pi_{2}=\frac{E(N)}{|J|}+\frac{E(N)}{2|J|}+0+0=\frac{3 E(N)}{2|J|}=1.125
\end{gathered}
$$

Clearly $\pi_{1}>\pi_{2}$, and thus Policy 10 does not satisfy entity desirability.

\section{F. ACTIVITY-BASED FAIRNESS}

For systems where the energy information might not be available, it is relevant to analyse whether the surrogate-based policies satisfy fairness properties based on the available information: activity. In this section we analyse the surrogate-based policies considering a variation of the fairness criteria where the antecedents of the properties are stated in terms of activity.

We define the activity-based fairness criteria translating the antecedent from energy to activity when possible:

Activity-based symmetry: if $a(S \cup\{i\})=a(S \cup\{j\})$ for all $S \subseteq N \backslash\{i, j\}$, then $\pi_{i}=\pi_{j}$.

Activity-based dummy player: if $a(S \cup\{i\})-a(S)=a(\{i\})$ for all $S \subseteq N \backslash\{i\}$, then $\pi_{i}=E(\{i\})$. Note that the apportionment policies divide the energy consumption, and thus the consequent of the property remains $\pi_{i}=E(\{i\})$ and not $\pi_{i}=a(\{i\})$, since the latter would imply that the apportionment policy apportions activity which is incorrect.

Activity-based individual rationality: if $I(E) \neq \emptyset$ and $\sum_{i \in N} a(i) \geq a(N)$ then $\pi_{i} \leq E(\{i\}), \forall i \in N$. $I(E) \neq \emptyset$ is a required precondition for an apportionment that satisfies $\pi_{i} \leq E(\{i\}), \forall i \in N$ and it is well 
known that $I(E) \neq \emptyset$ iff $\sum_{i \in N} E(\{i\}) \geq E(N)$. Thus, an individually rational apportionment has still requirements in terms of energy given the consequent.

Activity-based entity desirability: $i \succeq_{a} j \Longrightarrow \pi_{i} \leq \pi_{j}$, where $i \succeq_{a} j$ is defined as $a(S \cup\{i\}) \leq$ $a(S \cup\{j\}) \forall S \subseteq N \backslash\{i, j\}$.

\section{F.1 Activity-based symmetry}

We provide the proofs that show that Policy 8 and 9 satisfy activity-based symmetry, whereas Policy 10 does not satisfy the property.

Theorem F.1. Policy 8 (Proportional to activity) always satisfies activity-based symmetry.

Proof. In the proof we will assume $a(S \cup\{i\})=a(S \cup\{j\})$ for all $S \subseteq N \backslash\{i, j\}$, and show $\pi_{i}=\pi_{j}$ for Policy 8.

From Equation 10 the apportionment for entities $i$ and $j$ is the following:

$$
\begin{aligned}
& \pi_{i}=a(\{i\}) \frac{a(N)}{\sum_{k \in N} a(\{k\})} \\
& \pi_{j}=a(\{j\}) \frac{a(N)}{\sum_{k \in N} a(\{k\})}
\end{aligned}
$$

It follows from the antecedent of activity-based symmetry that $a(i)=a(j)$ for $S=\emptyset$. From Equation 10 and $a(i)=a(j)$ we have that:

$$
\pi_{i}=a(i) \frac{a(N)}{\sum_{k \in N} a(k)}=a(j) \frac{a(N)}{\sum_{k \in N} a(k)}=\pi_{j}
$$

Policy 8 satisfies symmetry.

Theorem F.2. Policy 9 (Time slicing) always satisfies activity-based symmetry.

Proof. In the proof we will assume $a(S \cup\{i\})=a(S \cup\{j\})$ for all $S \subseteq N \backslash\{i, j\}$, and show $\pi_{i}=\pi_{j}$ for Policy 9.

From Equation 11 the apportionment for entities $i$ and $k$ is computed using the function $f(i, j)$ which has two cases. We analyse each case separately.

(1) If $\sum_{i \in N} a(i, j)=0$, then $f(i, j)=f(k, j)=\frac{1}{|N|}$ for entities $i$ and $k$.

(2) If $\sum_{i \in N} a(i, j) \neq 0$, then $f(i, j)$ and $f(k, j)$ are the following:

$$
\begin{aligned}
f(i, j) & =\frac{a(i, j)}{\sum_{m \in N} a(m, j)} \\
f(k, j) & =\frac{a(k, j)}{\sum_{m \in N} a(m, j)}
\end{aligned}
$$

It follows from the antecedent $a(S \cup\{i\})=a(S \cup\{j\})$ for all $S \subseteq N \backslash\{i, j\}$ that $a(i, j)=a(k, j)$. From Equation 10 and $a(i, j)=a(k, j)$ we have that:

$$
f(i, j)=\frac{a(i, j)}{\sum_{m \in N} a(m, j)}=\frac{a(k, j)}{\sum_{m \in N} a(m, j)}=f(k, j)
$$

Since $f(i, j)=f(k, j)$ we have that $\pi_{i}=\pi_{k}$, and thus Policy 9 satisfies symmetry.

TheOREM F.3. Policy 10 (Last active) does not always satisfy activity-based symmetry.

Proof. A counterexample is enough to prove the theorem. We show that $a(S \cup\{i\})=a(S \cup\{j\})$ for all $S \subseteq N \backslash\{i, j\}$ holds in the conterexample and show $\pi_{i} \neq \pi_{j}$ for Policy 10 .

Figure 9 shows the counterexample for the system $N=\{1,2\}$ and the placement of the activity of each entity in time for the period $\left.T=\left[t_{1}, t_{5}\right]: s_{1}=\left[\left(t_{1}, 1\right),\left(t_{3}, 0\right)\right], s_{2}=\left[\left(t_{2}, 1\right),\left(t_{4}, 0\right)\right)\right]$, where $t_{1}=0, t_{2}=3$, Authors' preprint 
$t_{3}=4, t_{4}=7$ and $t_{5}=10$. There are four intervals where all entities are inactive in the last interval, i.e., $\sum_{i \in N} a(i, 4)=0 . l(2,4)=1$ since entity is the last active entity for interval 4 . For simplicity $E(N, j)=\frac{E(N)}{|J|}$ for the four intervals $j \in J(|J|=4)$ and $E(N)>0$.

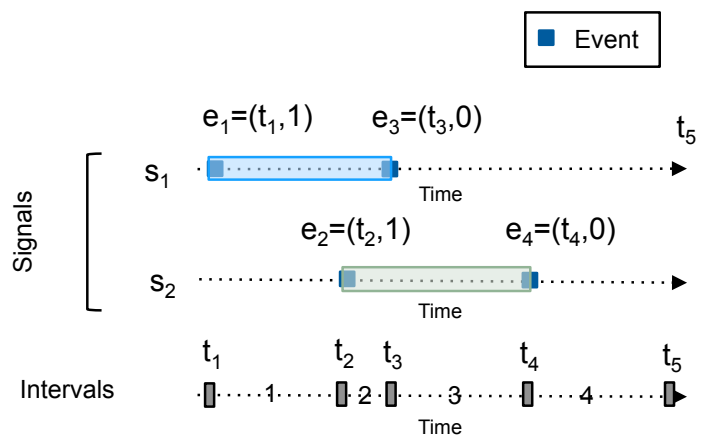

Fig. 9: Counterexample used in the proofs for activity-based symmetry for Policy 10.

The antecedent for the activity-based symmetry property holds since $a(1)=a(2)=4$. The apportionment for entities 1 and 2 is computed from Equation 12:

$$
\begin{gathered}
\pi_{1}=\frac{E(N)}{|J|}+\frac{E(N)}{2|J|}+0+0=\frac{3 E(N)}{2|J|} \\
\pi_{2}=0+\frac{E(N)}{2|J|}+\frac{E(N)}{|J|}+\frac{E(N)}{|J|}=\frac{5 E(N)}{2|J|}
\end{gathered}
$$

Clearly $\pi_{1} \neq \pi_{2}$, and thus Policy 10 does not satisfy activity-based symmetry.

\section{F.2 Activity-based dummy player}

Theorems B.3, B.4 and B.5 show that Policies 8, 9 and 10 do not satisfy the activity-based dummy player since the counterexamples show $a(S \cup\{i\})-a(S)=a(\{i\})$ for all $S \subseteq N \backslash\{i\}$ and prove $\pi_{i} \neq E(\{i\})$ for $i=2$.

\section{F.3 Activity-based individual rationality}

Theorems C.4, C.5 and C.6 show that Policies 8, 9 and 10 do not satisfy the activity-based individual rationality since in the counterexamples we assume $I(E) \neq \emptyset$ and $\sum_{i \in N} a(i) \geq a(N)$, and show that $\pi_{i} \leq$ $E(\{i\})$ is not satisfied for all $i \in N$.

\section{F.4 Activity-based entity desirability:}

We provide the proofs showing that Policies 8 and 9 satisfy activity-based entity desirability and Policy 10 does not satisfy the property.

Theorem F.4. Policy 8 (Proportional to activity) always satisfies activity-based entity desirability.

Proof. In order to prove the theorem we assume $i \succeq j$ and prove $\pi_{i} \leq \pi_{j}$ for Policy 8 .

It follows from the antecedent of activity-based entity desirability that $a(i) \leq a(j)$ for $S=\emptyset$. From Equation 4 and $a(i) \leq a(j)$ we have the following for $i$ and $j$ :

$$
\begin{aligned}
a(i) \frac{E(N)}{\sum_{k \in N} a(k)} & \leq a(j) \frac{E(N)}{\sum_{k \in N} a(k)} \\
\pi_{i} & \leq \pi_{j}
\end{aligned}
$$


Hence, Policy 8 satisfies entity desirability.

Theorem F.5. Policy 9 (Time slicing) satisfies activity-based entity desirability.

Proof. In the proof we will assume $a(S \cup\{i\}) \leq a(S \cup\{j\})$ for all $S \subseteq N \backslash\{i, j\}$, and prove $\pi_{i} \leq \pi_{j}$ for Policy 9.

From Equation 11 the apportionment for entities $i$ and $k$ is computed using the function $f(i, j)$ which has two cases. We analyse each case separately.

(1) If $\sum_{i \in N} a(i, j)=0$, then $f(i, j)=f(k, j)=\frac{1}{|N|}$ for entities $i$ and $k$.

(2) If $\sum_{i \in N} a(i, j) \neq 0$, then $f(i, j)$ and $f(k, j)$ are the following:

$$
\begin{aligned}
& f(i, j)=\frac{a(i, j)}{\sum_{m \in N} a(m, j)} \\
& f(k, j)=\frac{a(k, j)}{\sum_{m \in N} a(m, j)}
\end{aligned}
$$

It follows from the antecedent $a(S \cup\{i\}) \leq a(S \cup\{j\})$ for all $S \subseteq N \backslash\{i, j\}$ that $a(i, j) \leq a(k, j)$. From Equation 10 and $a(i, j) \leq a(k, j)$ we have that:

$$
\begin{aligned}
\frac{a(i, j)}{\sum_{m \in N} a(m, j)} & \leq \frac{a(k, j)}{\sum_{m \in N} a(m, j)} \\
f(i, j) & \leq f(k, j)
\end{aligned}
$$

Since $f(i, j) \leq f(k, j)$ for all $j$ intervals we have that $\pi_{i} \leq \pi_{k}$. Thus, Policy 9 satisfies symmetry.

Theorem F.6. Policy 10 (Last active) does not always satisfy activity-based entity desirability.

Proof. A counterexample is enough to prove the theorem. We show that $i \succeq j$ holds in the counterexample and prove $\pi_{i}>\pi_{j}$ for Policy 10 .

Figure 10 shows the counterexample for a system $N=\{1,2\}$ and the placement of the activity of the entities in time in the period $\left[t_{1}, t_{5}\right]: s_{1}=\left[\left(t_{1}, 1\right),\left(t_{3}, 0\right)\right], s_{2}=\left[\left(t_{2}, 1\right),\left(t_{4}, 0\right)\right]$, where $t_{1}<t_{2}<t_{3}<t_{4}<t_{5}$ and $\left(t_{3}-t_{1}\right)>\left(t_{4}-t_{2}\right)$. There are four intervals and all the entities are inactive in the fourth interval, i.e., $\sum_{i \in N} a(i, 4)=0$. Thus, $l(1,4)=0$ and $l(2,4)=1$. For simplicity $E(N, j)=\frac{E(N)}{|J|}$ for the four intervals $j \in J$ $(|J|=4)$.

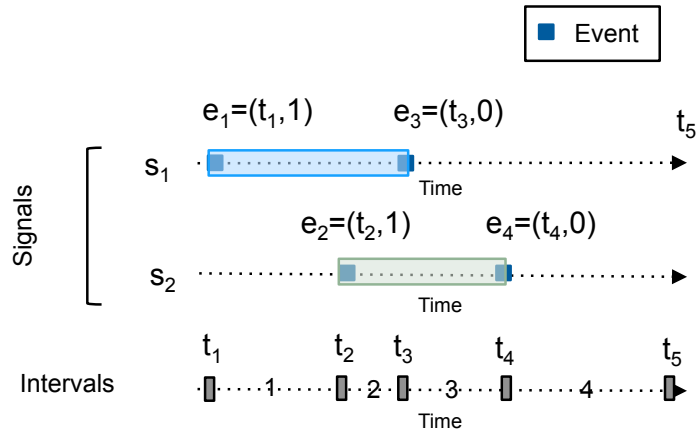

Fig. 10: Counterexample showing that Policy 10 does not satisfy entity desirability.

Since $\left(t_{3}-t_{1}\right)>\left(t_{4}-t_{2}\right)$ we have that $a(2)<a(1)$. Thus $2 \succeq 1$ holds in the counterexample.

From Equation 12 the apportionment for the entities is computed: 


$$
\begin{gathered}
\pi_{1}=\frac{E(N)}{|J|}+\frac{E(N)}{2|J|}+0+0=\frac{3}{8} E(N) \\
\pi_{2}=0+\frac{E(N)}{2|J|}+\frac{E(N)}{|J|}+\frac{E(N)}{|J|}=\frac{5}{8} E(N)
\end{gathered}
$$

Clearly $\pi_{1}<\pi_{2}$ even if $2 \succeq 1$, and thus Policy 10 does not satisfy activity-based entity desirability. 